\title{
MODELS OF COGNITION AND AFFECT IN PERCEPTIONS OF WELL-BEING ${ }^{1}$
}

(Received 17 May, 1979)

\begin{abstract}
How do people arrive at assessments of their own life quality? A series of models was developed to provide an interpretation of the way the factors of cognition and affect operate along with evaluations of specific life concerns (domains) in the perception of well-being. Following previous research, cognition was defined operationally as a factor which accounts for the covariance among a set of assessments of life-as-awhole after affect, as measured by Bradburn's scales, is partialled out and after allowance is made for the presence of correlated measurement errors. It was found that loadings on the cognitive factor, and hence the interpretation of this factor, changed little despite quite large changes in the models. Moreover, in all major comparisons, models that contained the cognitive factor fitted the data better than models that did not. Models that included affect as the only variable intervening between the domains and the life-as. a-whole factor led to results that were intuitively difficult to accept. In the preferred model both affect and cognition were positioned as intervening variables. In this model it was found that the domain evaluations had no direct impact on life-as-a-whole assessments - the contribution of the domains was indirect by way of their association with cognition and affect. It was hypothesised that associated with each domain was a domainspecific element of affect and a domain-specific element of cognition. The linear additive relation found by previous researchers between domain evaluations and life-as-a-whole assessments would then be explainable as a statistical result arising from the summing of the domain-specific elements of affect and cognition.
\end{abstract}

\section{INTRODUCTION}

This paper follows an earlier one (McKennell, 1978) which reviewed the general characteristics and implications of the distinction between cognition and affect in perceptions of well-being.

Cognition refers here to the process whereby relativism enters into satisfaction judgements. Satisfactions, it is commonly held, are tied to the expectations and standards of comparison in terms of which current circumstances are being evaluated. It is possible, for example, for people in relatively disadvantaged circumstances, because of their lower expectations, to report higher than average levels of satisfaction, and for the more privileged, because of their higher expectations, to be dissatisfied. Affect, on the other hand, refers to the individual's immediate feeling state which is not anchored, or not tied to the same extent, to cognitive frames of reference.

This kind of distinction is sometimes made, at a semantic level, between 'happiness' and 'satisfaction'. The scales developed by Bradburn (1969) to 
measure affect $d o$ correlate more highly with happiness than with life-satisfaction ratings, but only marginally.

The earlier paper (McKennell, 1978) posited and provided some evidence to show that currently used rating-scale indicators of perceived well-being are compounds of both affect and cognition, differing only marginally in the extent to which they depend on one or the other factor. One analysis was reported, for example, which focussed on the area of non-overlap between happiness and life-satisfaction ratings by dichotomising and cross-tabulating the two indicators and then observing what further variables changed diagnostically between appropriate cells of the $2 \times 2$ table. Differential trends with age and education, barely discemible in straightforward correlations against the indicators, then emerged strongly along with further interesting differences for other outside variables.

In addition, a second kind of analysis was reported which forms the basis of the present paper. These analyses drew on data sets, regrettably few, which included Bradburn's affect scales and also a range of global ratings of subjective well-being. Bradburn's (1969) affect scales were taken as the best available measures of affect. Although no direct or even proxy indicators of cognition were available, the considerable variance in the global ratings which remained after the affect scales were partialled out and allowance was made for correlated method effects was attributed to cognition. Starting points were then explored for analysing the role of cognition and affect more exactly by means of path models which included the cognitive factor as an unobserved variable.

The present paper continues this line of investigation. A related paper (Andrews and McKennell, 1978) uses a similar approach to develop empirical estimates of the percentage of variance in certain measures of perceived wellbeing that reflect cognitive, affective, or other (mainly random or correlated error) factors.

\section{PROCEDURE}

\subsection{Analytical Strategy and Scheme of the Present Paper}

Our research objective is to construct a model which will not only include a cognitive factor but will provide an interpretation of the way this factor 
operates along with affect and evaluations of specific concerns in the perception of global well-being or life-as-a-whole (LAW). In such a model the LAW factor will be positioned as an endogenous variable and the flow of determining influences from other factors will be portrayed as cause-effect relationships. Because it seeks to specify causal paths between factors, this kind of model may be referred to, briefly, as a 'factor-path' model.

Such a model can be constructed in two stages. In the first stage we can develop a path model which contains factors as unobserved or unmeasured variables but does not interpret the relationships between them. The factors in this first stage are left as exogenous unobserved variables which occur only as the unanalysed causes of the observed variables. The factors may or may not be correlated. At the second stage an interpretation of the association between the factors in terms of causal influences is attempted.

It makes good sense to proceed in the two-stage fashion in dealing with the factor of cognition. Cognition is an unmeasured, i.e., unobserved, variable in the sense that we do not have even indirect or proxy measures available. It is theory together with the observed pattern of relationships among the other variables in the system which give the cognitive factor its meaning. There would be no point in attempting to construct factor-path models that include cognition unless it could be demonstrated that (1) a factor of cognition can be given consistent meaning and (2) is necessary to explain the covariation among the set of observed variables. This demonstration is made in Section 3 of the present paper.

Section 4.2 examines factor-path models that do not include cognition, and attempts to develop them as far as they will go. The consequences of adding in the cognitive factor are then examined in Section 4.3. An evaluation of factor-path models with and without a cognitive factor is then made in Section 4.4. Section 5 considers some parallel analyses on other data sets. Section 6 summarises and discusses the results.

\subsection{Statistical Techniques}

Since cognition is available to us only as a residualised factor and we wish to study its role in the flow of influences leading to life quality assessments, we were led naturally to methods involving path models and factor analytic techniques. It has been shown that such methods are special cases of structural equation models (Goldberger, 1972; Goldberger and Duncan, 1973). 
Joreskog $(1969,1970,1973)$ has developed a powerful maximum-likelihood technique for simultaneously estimating parameters for observed and unobserved variables in a structural model that allows error components to be correlated.

Model estimation. This method, as embodied in the LISREL computer program (Joreskog and Sorbom, 1976), provided the principal tool for the analyses reported throughout this paper. In their description of the LISREL model, Joreskog and Sorbom note that it

consists of two parts: the measurement model and the structural equation model. The measurement model specifies how the latent variables or hypothetical constructs are measured in terms of the observed variables and is used to describe the measurement properties (validities and reliabilities) of the observed variables. The structural equation model specifies the causal relationships among the latent variables and is used to describe the causal effects and the amount of unexplained variance.

By imposing various restrictions on the more general model, the LISREL program can easily be made to estimate the measurement model on its own. ${ }^{2}$ LISREL therefore provided us with a ready means for carrying through the two-stage strategy outlined above. The results in Section 3 were obtained using the measurement model. What we have termed factor-path models were obtained in Section 4 utilising the more general features of the program.

As noted above it was essential for our operational definition of cognition that the covariance remaining among global indicators after affect was removed should also be shorn of correlated errors due to method effects. The capacity of the LISREL program to handle correlated errors was therefore of prime importance for the analytical work. Considerable effort was expended in obtaining the best possible estimates for the method effects. This part of the development work is reported in a separate paper (Andrews and McKennell, 1978). For most of the analyses reported in this paper method-effect parameters were constrained to equal the previously estimated values while we concentrated on other features of the model.

Specification of the program input for LISREL is considerably facilitated if the model under examination is first drawn out in full detail as a path diagram. A few key models out of the many examined are shown as diagrams below. The diagrams are much more than computing aids. They share the property of all properly constituted path diagrams of "being isomorphic with the algebraic and statistical properties of the postulated system of variables" (Duncan, 1966). There is a one-to-one correspondence between the postulated causal and noncausal relationships of the model under investigation and its 
path diagram. In the process of specifying the diagrams, one is forced to be explicit about the properties being assumed for the model. This makes it easier to spot from the program output the reasons why a particular model is producing a relatively poor fit to the data and to think of improvements.

Path analysis itself assumes that certain basic properties hold for the data, namely that the measures approximate interval scales and the relationships are tolerably free from curvilinearities and interactions. Previous work has shown that data from perceived quality of life surveys conform reasonably well to these assumptions (see Andrews and Withey, 1976, pp. 116-117; Campbell et al., 1976, pp. 77-80).

Choosing among models. The computer program used to estimate the models includes a routine for calculating the fit of a model to the data. Unfortunately, for the relatively large sample sizes involved in our research, chi-square is not useful as a precise 'test' statistic for differentiating between theoretical structures. Save in limited circumstances, ${ }^{3}$ accept-reject decisions associated with a statistical probability level of chi-square are not feasible. But chi-square can be used as a qualitative or descriptive statistic for exploratory purposes. In comparing two models, the one with more fitted parameters, and therefore fewer degrees of freedom, usually yields a smaller chi-square.

\begin{abstract}
A large drop in chi-square, compared to the difference in degrees of freedom, indicates that the changes made in the model represent a real improvement. On the other hand, a drop in chi-square close to the difference in number of degrees of freedom indicates that the improvement in fit is obtained by 'capitalizing on chance,' and the added parameters may not have real significance and meaning (Joreskog and Sorbom, 1976, p. 12).
\end{abstract}

This qualitative or descriptive use of chi-square can therefore serve as an aid in assessing the fit of a particular model in relation to alternatives. Occasionally in moving from a more complex to a simpler model with fewer estimated parameters we found that chi-square actually decreased even though the degrees of freedom had increased (see for example Exhibit 7), and in these instances the chi-square comparison did seem to provide firm evidence for the improved fit of the simpler model. But in general we have not relied exclusively on statistical criteria of goodness-of-fit but have weighed these against the substantive implications of alternative models. As Joreskog $(1969$, p. 201) has written,

When to stop fitting additional parameters cannot be decided on a purely statistical basis. This is largely a matter of the experimenter's interpretations of the data based on substantive theoretical and conceptual considerations. Ultimately the criteria for goodness of the model depends on the usefulness of it and the results it produces. 
For comparisons among what we have termed factor-path models a second statistical dimension of adequacy becomes available. In these models one or more factors are serving as theoretically endogenous variables. The life-as-awhole factor (LAW), for example, always occupies this position in our factorpath models. Such models can be compared with respect to the extent to which the variance in the theoretically endogenous variable is being predicted by the other variables in the proposed structure. The LISREL program computes an estimate of the residual or unexplained variance for each endogenous variable in a structural equation model. This parameter is entirely analogous to the squared residual path coefficient in path analysis with observed variables. It is equal to $1-R^{2}$, where ' $R$ ' here refers to the multiple correlation of a particular endogenous unobserved variable with the causal antecedent unobserved variables. Models can therefore be compared with respect to the size of residual coefficients. Burt $(1973$, p. 145) has referred to this second dimension of adequacy as the 'external validity' of a model "since it is solely concerned with the ability of the structure as a source of prediction".

\subsection{The data analysed}

The analyses require data which include measures of global well-being - e.g., evaluations of life-as-a-whole; a set of evaluations of specific life concerns e.g., ratings of family life, community, income, privacy, the national government, etc.; plus Bradburn's (1969) affect scales. Three different data sets have been examined.

The bulk of the statistical work was carried out on one of the data sets in the Andrews-Withey series, namely their November 1972, Form 2 national survey. This was based on a representative sample of American adults and included 1072 respondents. Full details about the measures are given in Andrews and Withey (1976). Results from the analysis of these data appear in Section 3 and 4 of the present paper, with brief mention also being made in Section 5.

Data from a British survey were also analysed. This survey is described by Abrams (1976) and Hall (1976) and involved interviews in March 1975 with 932 respondents drawn from a representative sample of people aged 16 and over living in British metropolitan areas. These results are reported in Section 5.

The third data set was assembled from a series of American national surveys conducted by the National Opinion Research Center and is described in a 
paper by Burt, Wiley, Minor, and Murray (1978). Section 5 also includes a brief discussion of these results.

\section{FACTOR MODELS: AFFECT-COGNITION VERSUS AFFECT-LAW}

\subsection{Concepts and Procedures}

The essence of the comparison to be made in this section is conveyed in simplified geometric form in Exhibit 1.

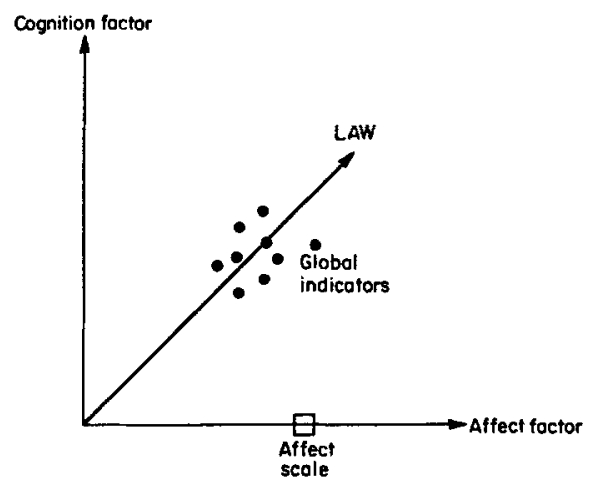

Exhibit 1. Positions of global indicators of life-as-a-whole (LAW) in the two dimensional space defined by cognition and affect (simplified results).

Although Exhibit 1 is not a plot of actual data it has been drawn to reflect the main findings. A handful of indicators of global well-being are shown as points plotted with respect to two orthogonal axes representing the affect and cognition factors. Following the usual conventions for the geometric representation of factors (Harman, 1976), a factor loading for an indicator is represented by its projection on one of the axes. Thus all indicators load on both affect and cognition to varying degrees and they cluster together in the factor space because they are fairly highly intercorrelated. Only one affect factor is shown for simplicity, its positon being fixed by an affect scale. There are no measured variables to define the cognitive factor directly, but its position has been fixed by making it orthogonal to the affect factor. Cognition is thereby defined as a factor which accounts for the covariance in the observed measures which remains after that due to affect is partialled out. 
The life-as-a-whole factor, LAW, is defined by the factor axis which passes through the centroid of the cluster of indicators. If all points fell on this axis, then only one factor would be needed to reproduce the observed correlations. To the extent that the points spread away from the LAW axis, two factors will fit the data better. The points are shown clustered fairly close to the LAW axis, and this suggests that this one factor will do a reasonable job of accounting for the observed correlations. But there is some scatter around the line, and hence the fit to the observed data will be somewhat better with a two factor solution. This is the essence of what was found in the analyses to be described below.

Before these analyses are introduced, two further observations are necessary. If the same measurement method is employed for the global indicators, then some part of their covariation will arise from that fact alone. The covariance that remains after affect is partialled out would thus be due to both a method factor and to cognition. It follows that a method factor must be introduced into the analysis. Cognition will then be defined as the factor which accounts for the covariance among a set of global indicators after affect is partialled out and after allowance has been made for the presence of correlated error. For the sake of simplicity, the figure in Exhibit 1 omits this method factor.

Exhibit 1 simplifies the actual analysis in another way. The figure shows just a single affect factor whereas Bradburn's (1969) work suggests the necessity of considering two distinct types of affect: Positive affect and Negative affect. A separate scale for each type of affect is included in our analysis, and these two types of affect have been included in our models as two distinct factors.

A comparison of the goodness-of-fit of factor models can be made by means of confirmatory factor analysis using the LISREL program. Exhibits $2 a$ and $2 b$ show the details of the factor models being compared.

While the geometric representation in Exhibit 1 above was helpful in conveying the basic notions, it is not adequate for describing the details of the analytic work. The representation in Exhibit 2 and throughout the remainder of this paper will be in terms of causal path diagrams. The assumptions of the model are mapped precisely in the diagrams. The relationship between a factor (shown as an oval) and an observed variable (shown as a rectangle) is represented as a causal path, the path coefficient being equal to the size of the factor loading. (All coefficients are reported in standardized form -i.e., 


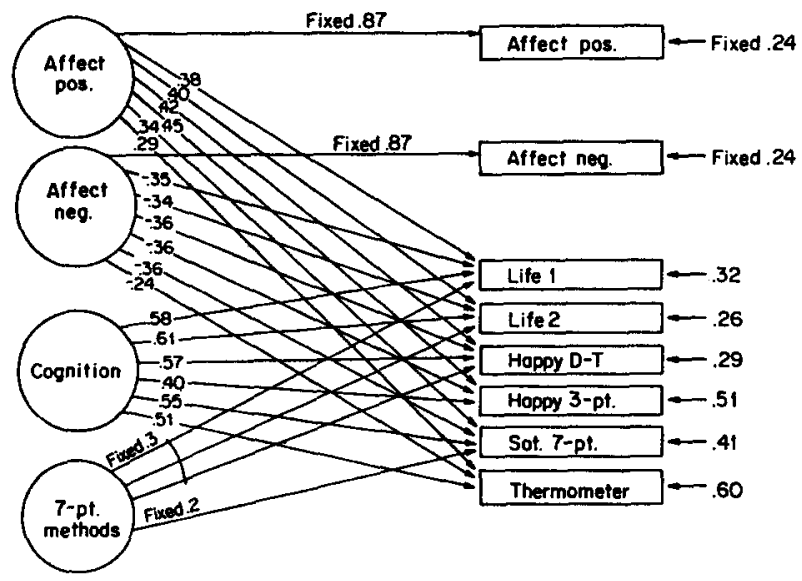

LISREL fit stotistics: $\mathrm{Chi}^{2}=23, \mathrm{df}=12, \mathrm{~N}=1072$

All exogenous foctors statistical ly independent.

Exhibit 2a. Factor model explaining global indicators by affect and cognition while allowing for random and correlated measurement errors.

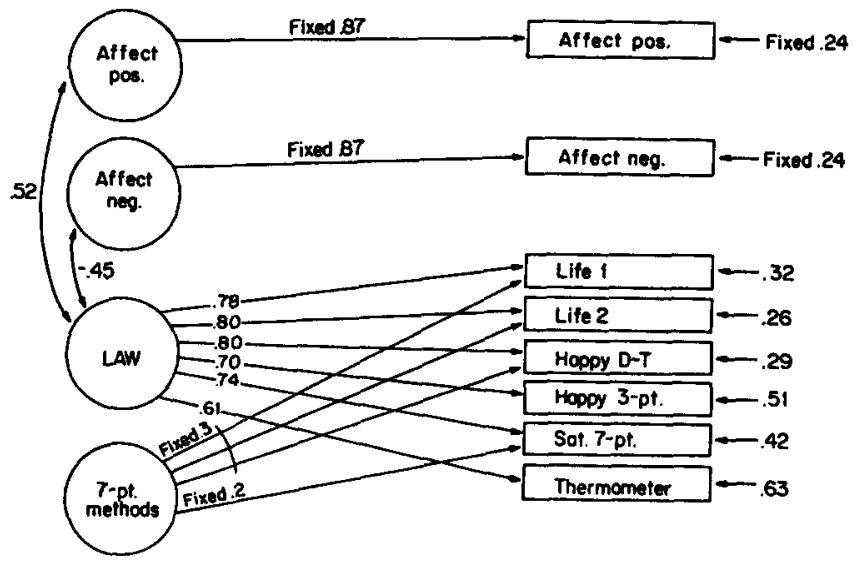

LISREL fit stotistics: $\mathrm{Chi}^{2}=62, \mathrm{df}=22, \mathrm{~N}=1072$.

Exhibit 2b. Factor model explaining global indicators by affect and life-as-a-whole (LAW) while allowing for random and correlated measurement errors.

after transforming all variables and factors to have variances of 1.0.) The absence of a path implies a restriction built into the model: that the linkage has been fixed at zero. Unanalysed correlational associations are shown by double-headed arrows. 
The model assumes that the variance in the global indicators derives from three sources: the true effect of the underlying factor, a method effect giving rise to correlated errors, and a residual or random error component. Accordingly the parameter estimates associated with the linkages between a measured variable and either a wellbeing or a method factor are interpreted as validity or method effect coefficients, respectively. The parameter values shown in Exhibit $2 \mathrm{a}$ and $2 \mathrm{~b}$ are the estimates computed by the LISREL program. Also shown are the computed chi-square values which are based on how well the constrained and free parameters that comprise the model reproduce the observed correlations. As discussed earlier, the change in chi-square in relation to the change in degrees of freedom provides a means of comparing two models in terms of their fit to the observed data.

A model that includes global indicators and cognitive as well as affect factors, as in Exhibit 2a, can be regarded in two ways. In the first place, it can be seen as a way of partitioning the variance of each global indicator into a part due to cognition and parts due to positive and negative affect. This topic has been addressed in a separate paper (Andrews and McKennell, 1978). The present paper focusses on the second way in which the model in Exhibit 2a can be regarded, namely as a representation of the structure underlying perceptions of well-being. From this perspective Exhibit 2a can be regarded as one hypothesis to be compared with an alternative hypothesis embodied in Exhibit $2 \mathrm{~b}$. The latter model covers the same set of observed variables but dispenses with the cognitive factor and substitutes a life-as-a-whole factor. Exhibit $2 b$ thus attempts to account for the covariance among the global indicators, other than Bradburn's affect scales, by only one substantive factor. Apart from method effects, the global indicators are represented as tapping only this one underlying factor, life-as-a-whole (LAW), and any correlation they have with the affect measure is held to come about indirectly through the correlation between the LAW factor and the affect factor. In what follows, models of the former type (Exhibit 2a) will be referred to as 'affectcognition' models, while those like that in Exhibit $2 b$ will be referred to as 'affect-LAW' models. The affect-LAW models leave unanalysed the possibility that the original evaluations may be compounded of separate cognitive and affective factors. The hypothesis that a cognitive factor plays a role in the perception of well-being can therefore be tested by seeing which type of model best fits the data. ${ }^{4}$ 


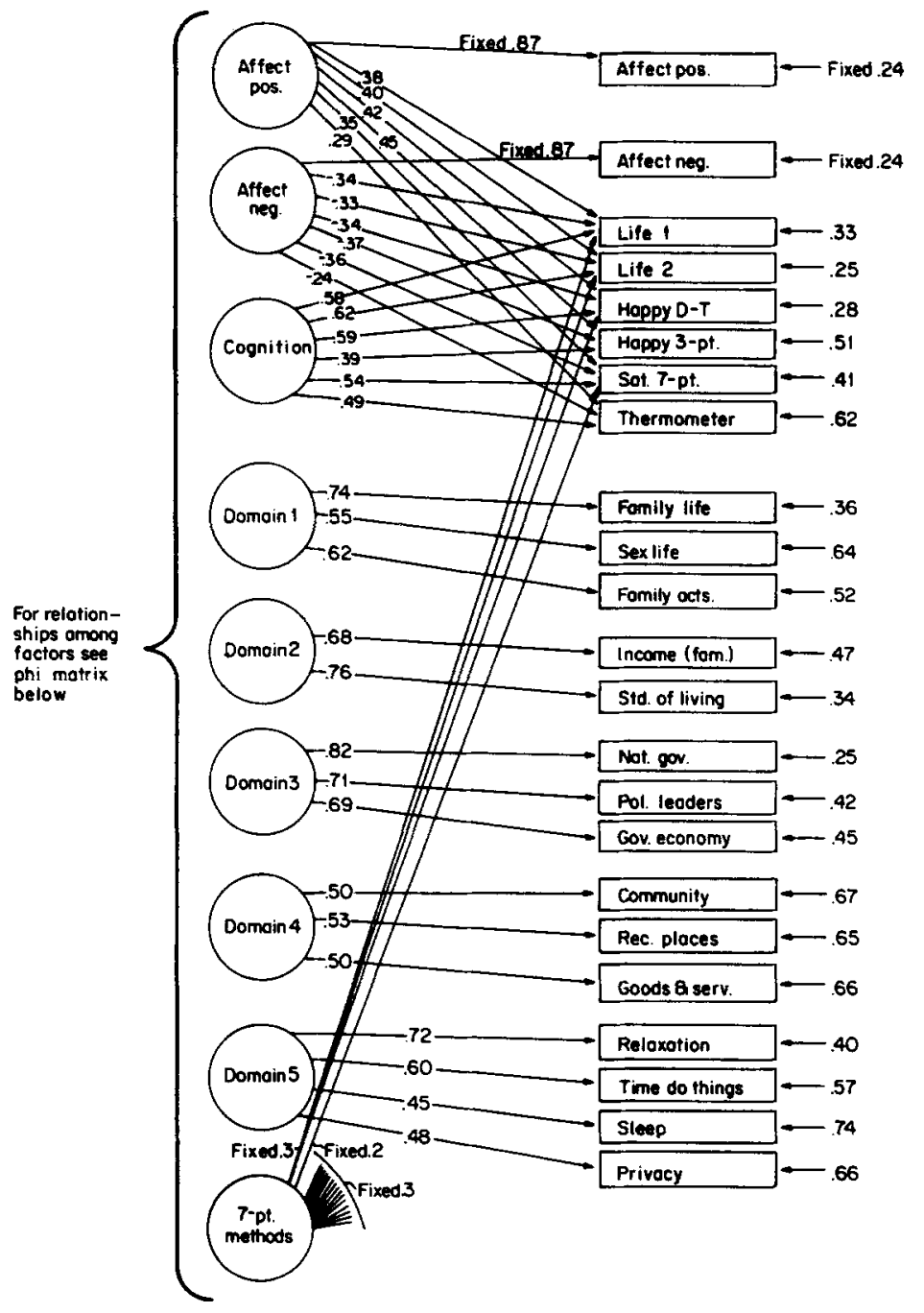

LISREL fit statistics: $\mathrm{Chi}^{2}=562$, of $=197, \mathrm{~N}=1072$

Estimated relationships ( $r$ 's) among exogenous foctors [ $f$; fixed]

\begin{tabular}{|c|c|c|c|c|c|c|c|c|}
\hline & $+A$ & $-A$ & $\operatorname{Cog}$ & D1 & D2 & 03 & $04^{\circ}$ & D5 \\
\hline Affect neg. & 6.00 & & & & & & & \\
\hline Cognition & 1.00 & 9.00 & & & & & & \\
\hline Somoin 1 & .47 & -.37 & .53 & & & & & \\
\hline Domain 2 & .39 & -37 & .46 & .56 & & & & \\
\hline Domain 3 & .19 & -.21 & .08 & .91 & .29 & & & \\
\hline Domain 4 & .22 & -.29 & .23 & .29 & .57 & .39 & & \\
\hline Domain 5 & $\begin{array}{r}.18 \\
+\infty\end{array}$ & $\begin{array}{r}-35 \\
t 00\end{array}$ & $\begin{array}{r}35 \\
100\end{array}$ & $\begin{array}{r}.49 \\
+00\end{array}$ & .51 & .22 & .50 & $+\infty$ \\
\hline
\end{tabular}

Exhibit 3a. Factor model explaining global indicators by affect and cognition in the presence of domains, while allowing for random and correlated measurement error. 


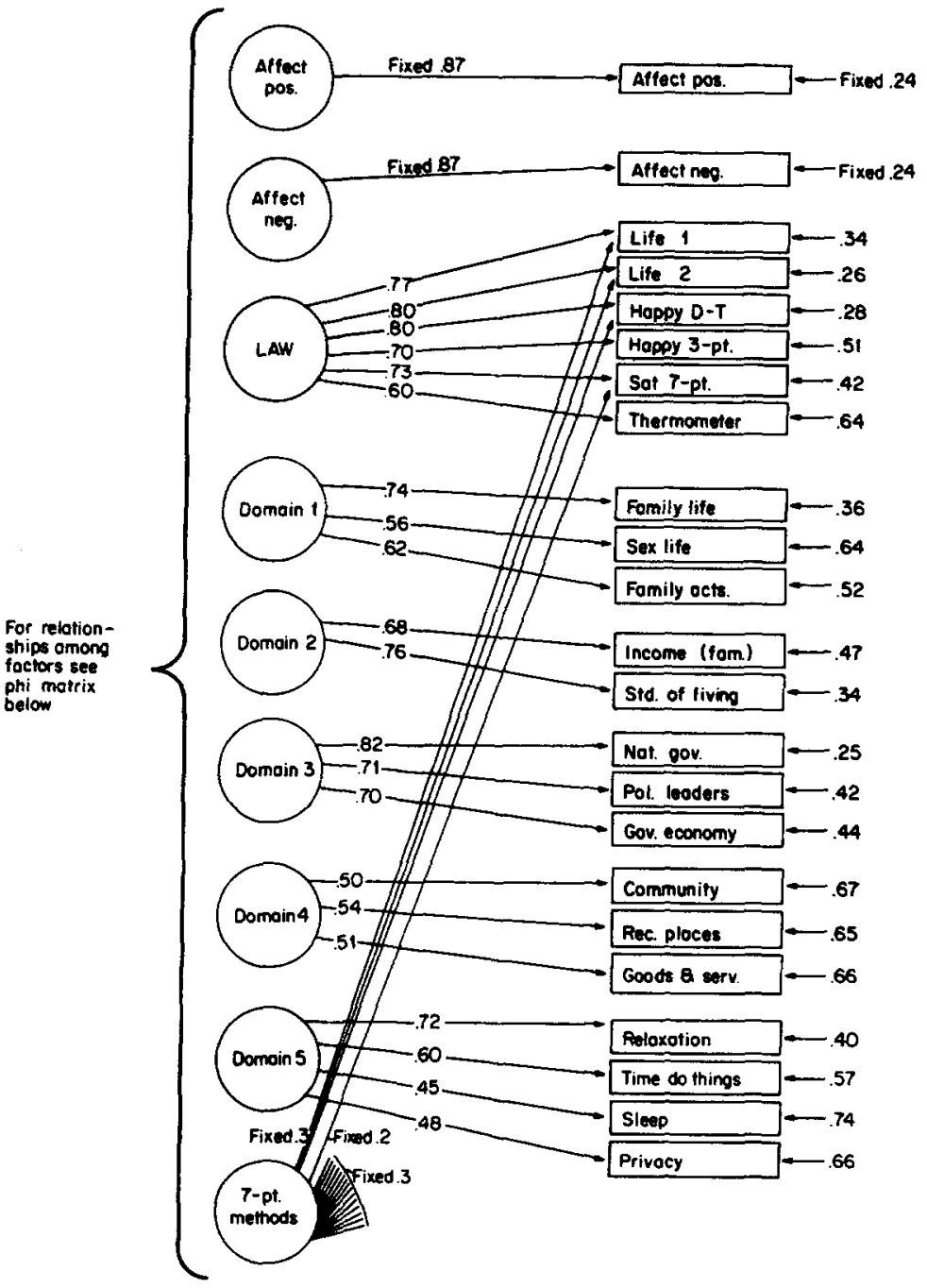

LISREL fit stotistics: $\mathrm{Chi}^{2}=603, \mathrm{df}=207, \mathrm{~N}=1072$.

Esfimoted relotionships ( $r$ 's ) among exogenous foctors [ $f=f i x e d]$

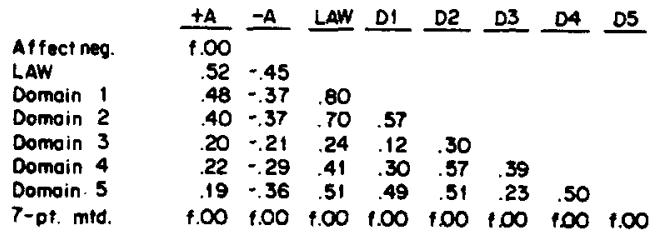

Exhibit 3b. Factor model explaining global indicators by affect and life-as-a-whole (LAW) in the presence of domains, while allowing for random and correlated measurement error. 


\subsection{Statistical Results: Affect-Cognition Compared with Affect-LAW}

The models in Exhibits $2 a$ and $2 b$ afford one contrast, the simplest we will be examining, between affect-cognition and affect-LAW models. An important finding is that the affect-cognition model has a smaller chi-square value in relation to its degrees of freedom than does the affect-LAW model. The difference in chi-squares (39.4) is substantially greater than the loss in degrees of freedom (10). The results indicate therefore that the affect-cognition model, Exhibit 2a, is here providing the better fit to the observed data. ${ }^{5}$

The structures for the perception of well-being that we consider below will include evaluations of specific life concerns as well as of global well-being. The contrast between the affect-cognition and affect-LAW models needs therefore to be extended to more complete models that include factors at the domain as well as the global level. ${ }^{6}$ This is done in the models in Exhibits 3a and $3 \mathrm{~b}$.

The 15 domain items used here were selected on the basis of earlier work on the factor analysis and perceptual mapping of much larger sets of domain evaluations (60-100) (see Chapter 2 and Appendix E, Andrews and Withey, 1976). The five factors used in Exhibit 3 were chosen, first, so that their constituent items would be well spread out in the perceptual space established by the perceptual mapping and, second, so that there would be a range in the strength of the correlations between the domain evaluations and the affect measures.?

The model in Exhibit 3a, which is an affect-cognition model, fits more parameters than that in Exhibit 3b, a LAW model, but the loss of 10 degrees of freedom is again small compared to the much larger reduction in chi-square (41). For these more complete structures, then, it can also be inferred that the affect-cognition model fits the data better than the affect-LAW model.

\subsection{Statistical Results: Affect-Cognition Combined with LAW}

Eventually, in Section 4, we will want to consider path models that make the LAW factor an endogenous variable dependent on both cognition and affect as well as domain evaluations. Therefore, the work in the present section needs to be still further extended to cover factor models that include all three global factors in addition to domain evaluations. This is done in the model in Exhibit 4. This model combines the features of the models in both Exhibits $3 \mathrm{a}$ and $3 \mathrm{~b}$. Three of the global indicators have been used to define 


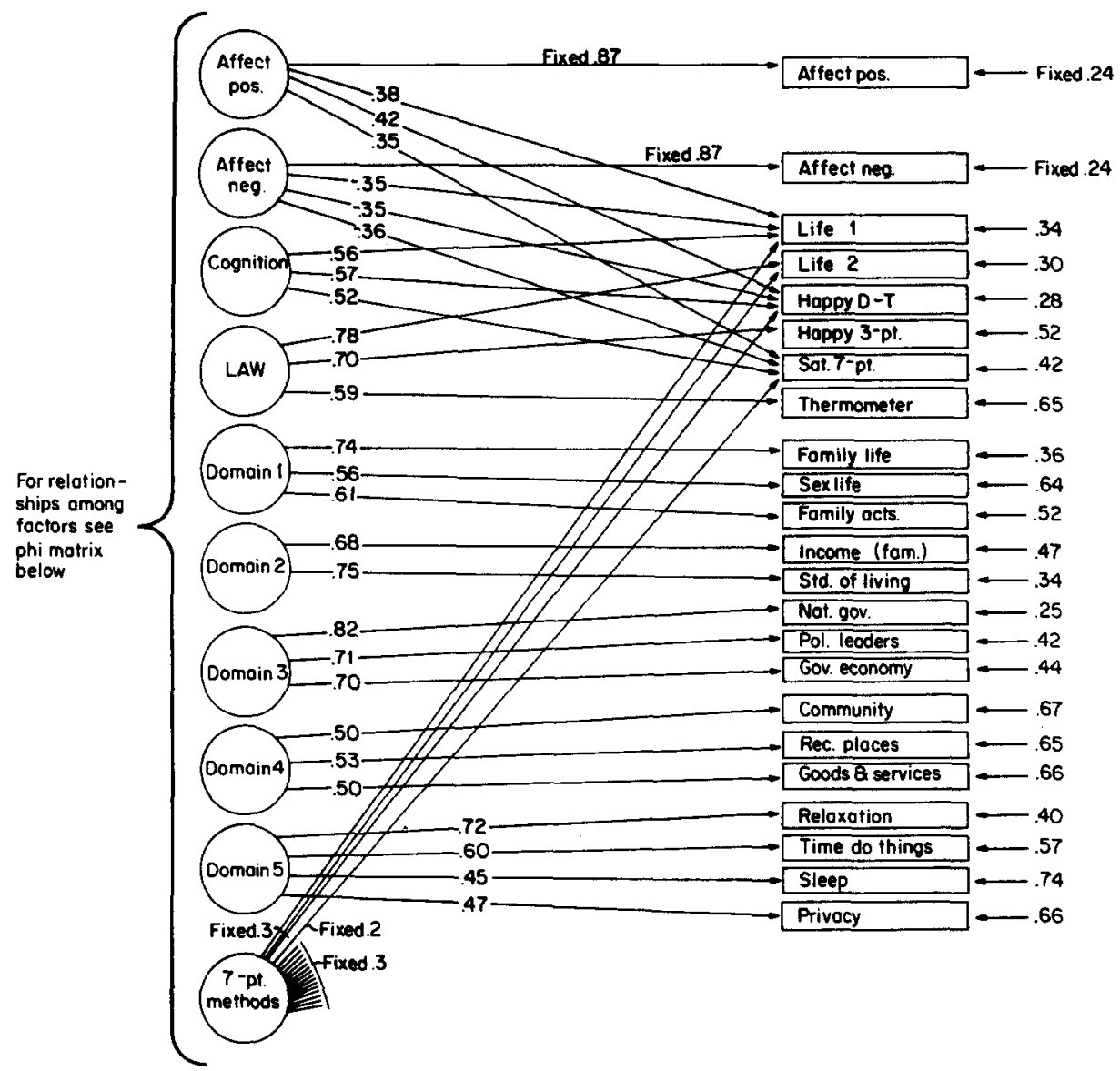

LISREL fit stotistics: $\mathrm{Chi}^{2}=574$, df $=196, \mathrm{~N}=1072$.

Estimoted relationships ( $r$ 's) omong exogenous factors [ $f$ = fiked]

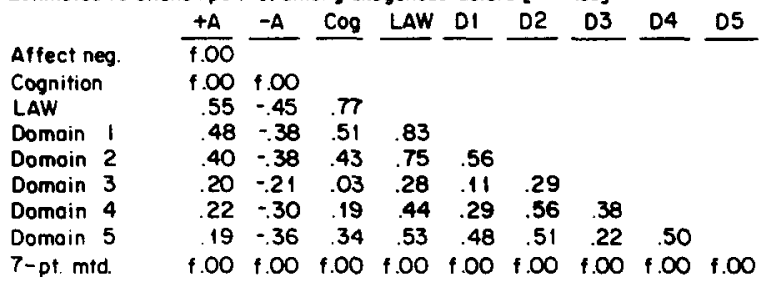

Exhibit 4. A model containing factors for both cognition and life-as-a-whole (LAW), as well as affect, domain, and method factors. 
the LAW factor and the remaining three to define a global cognition factor. The result can be considered either as a variant of the model of Exhibit $3 b$ into which a cognitive factor has been introduced, or of the model of Exhibit 3a into which a LAW factor has been included.

It is important to note that despite the added complexity, the parameter values in Exhibit 4 are consistent with those derived in the simpler models. The loadings of the three indicators defining global cognition are virtually identical with those in Exhibit 3a, and, except for the additional new correlations among the now expanded set of factors, all parameters in Exhibit 4 are also consistent with those in Exhibit 3b.

As is the case for all the models considered in this section, there is no implication about the dynamics underlying the relationships between the factors. The elaboration of the model in Exhibit 4 into a factor-path model will be the concern of Section 4.

A comparison of the reduction in chi-square relative to the drop in degrees of freedom suggests that, despite its added complexity, the model in Exhibit 4 still fits the data better than the affect-LAW model in Exhibit 3b, though not better than the affect-cognition model in Exhibit 3a. The main point about the Exhibit 4 model, though, is that it contains all the factors necessary for considering the role played by cognition and affect together with domain evaluations in the perception of well-being we needed to be satisfied with the properties of this structure before proceeding further.

\subsection{Comments}

An outstanding feature of the overall results in this section is that the movement from the simpler models, in Exhibit 2a and 2b, to those in Exhibits 3a and $3 \mathrm{~b}$, and then to the still more complex model in Exhibit 4 produced very little change in the loadings of the global indicators on either the LAW factor or the affect and cognition factors. The empirical meaning that can be assigned to these factors has thus remained constant even as models were expanded to encompass a wider range of observed variables, and as simpler models were integrated into more comprehensive structures. The constant meaning for the global factors of cognition, affects, and Life-as-a-whole in Exhibit 4 is particularly impressive.

At one point in the analytical work we wondered whether the results shown in Exhibit 4 were unduly influenced by the fact that the affect factors used to residualize global measures to produce cognition were in the direct 
circuit involving the LAW factor. We therefore ran a still more complex model (not shown) in which the affect measures were introduced twice, once to residualise cognition and quite separately to relate to LAW. All the parameter values turned out to be the same as in Exhibit 4. We find, therefore, that the separate parts of the more complex models are robust when taken on their own. The unobserved variables, the core concepts of the study, retain a consistent interpretation despite quite large changes in the models. This is an important consideration in the present study where one of these concepts, the cognitive factor, has no direct indicators but takes ist meaning from the structural relationship with other substantively different unobserved variables.

\section{FACTOR-PATH MODELS}

\subsection{Introduction}

In the foregoing section factors were discussed only as causes of the observed variables. Models that contained a cognitive factor were contrasted with models that did not. It was concluded that the cognitive models yielded a somewhat better fit to the observed data. We now consider models that embody hypotheses about the causal relations among the factors themselves. These models go beyond a purely statistical accounting for the variances in the observed measures. They are psychological as well as statistical models in that they suggest the underlying processes by which respondents may arrive at their evaluations of their own well-being. Once again models that contain a cognitive factor are to be contrasted with models that do not, but the objective now is to interpret the dynamics of the judgement process. As will be seen, we arrive at quite different interpretations according to which type of model we accept.

Section 4.2 describes the development of models that do not contain the cognition factor, and the following section 4.3 , considers models that do. The results are different and the contrasting implications for the judgement process are then discussed and evaluated in Section 4.4.

\subsection{Models Without a Cognitive Factor}

Exhibit 5 provides a convenient notation for the main features of the models to be discussed in this section. 


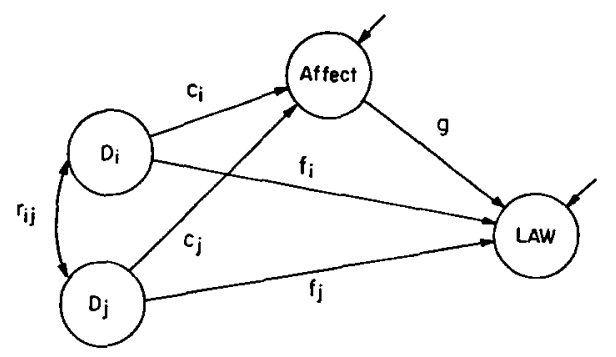

Exhibit 5. Basic form for path model with affect intervening between domains and life-as-a-whole (LAW).

Domain measures, $D_{\mathrm{i}}$ and $D_{j}$, are shown linked by paths $c_{i}$ and $c_{j}$ to affect (represented as a single variable for simplicity) and by paths $f_{i}$ and $f_{j}$ to a global measure, LAW (life-as-a-whole). The path from affect to the global measure is denoted by $g$.

Models of increasing complexity, but of this same general form, were examined, and the same two main outcomes always emerged. First, the path $g$ was always very small and could be eliminated without loss of explanatory power. Second, the $c$ paths need to be replaced by correlational links: that is, affect needs to be moved from its position as an endogenous variable intervening between the domains and LAW, and made exogenous on a par with the domains.

We will briefly describe the simpler analyses that gave these results before presenting in detail more developed models of this general type. Because a cognitive factor is not involved, models of the general type in Figure 5 can be constructed directly in terms of the observed variables. This type of model was constructed originally for the British data by Hall (1976). A replication on American data, and the implications for the $c$ and $g$ path was discussed briefly by McKennell (1978) and will be referred to again in Section 5 of the present paper. $^{8}$

Affect: Exogenous or endogenous? Exhibit 6 shows one version (we shall call it $6 \mathrm{~A}$ ) of the more elaborated model on which the basic theory was finally tested. It includes separate domain factors and a method factor, along with factors for affect and life-as-a-whole.

Four variants of this model - all of which are path analogues of the factor model shown in Exhibit 3 - were considered. The summary results - chisquared values, degrees of freedom, and the residual term $\left(1-R^{2}\right)$ for the prediction of LAW - are shown in Exhibit 7.

In the original model $-6 \mathrm{~A}$, Exhibit $6-$ the estimated values of the affect- 


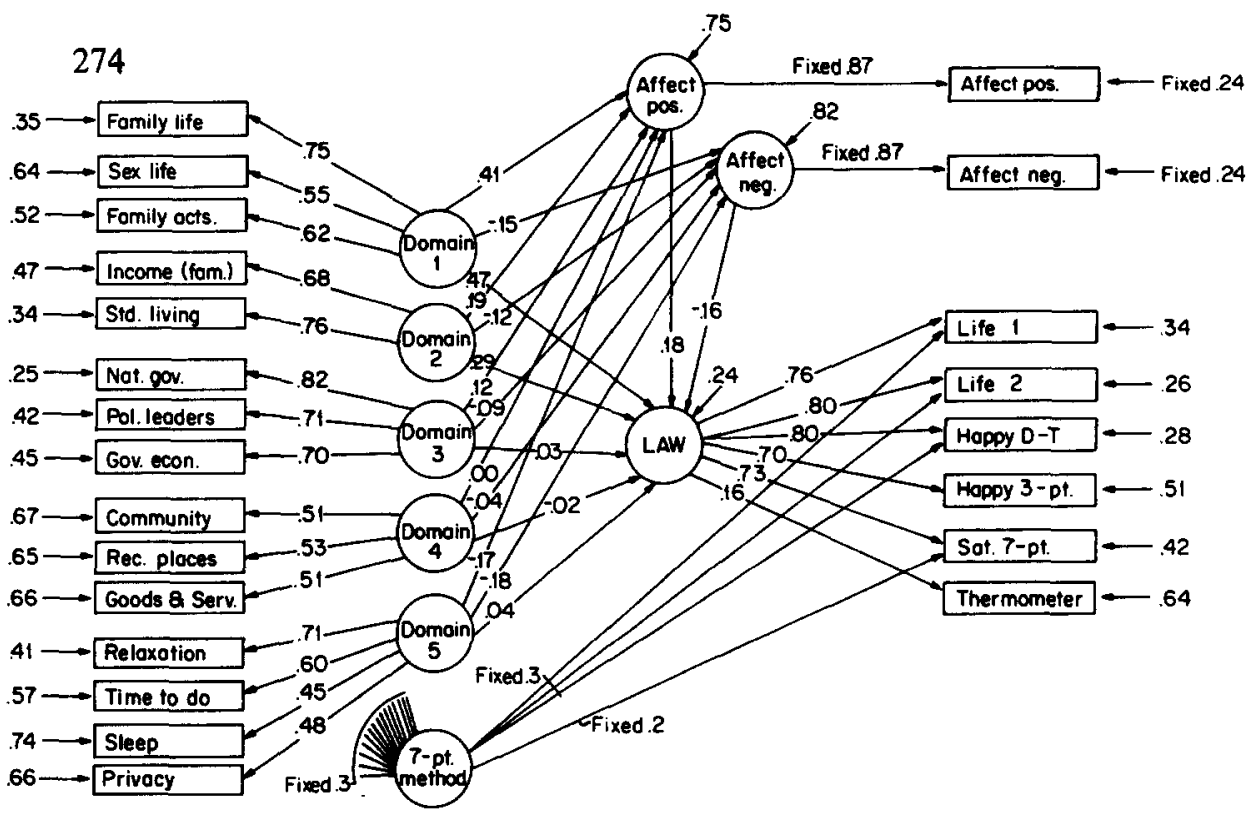

LISREL fit statistics: $\mathrm{Chi}^{2}=637$, of $=205, \mathrm{~N}=1072$

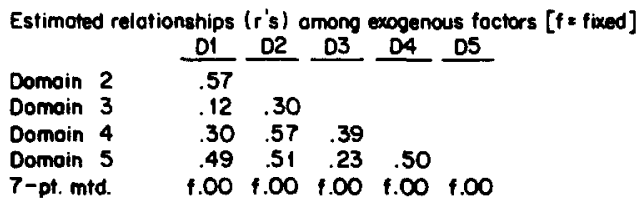

Exhibit 6. Factor-path model with affect intervening between domains and life-as-awhole (LAW), and with allowance for random and correlated measurement errors.

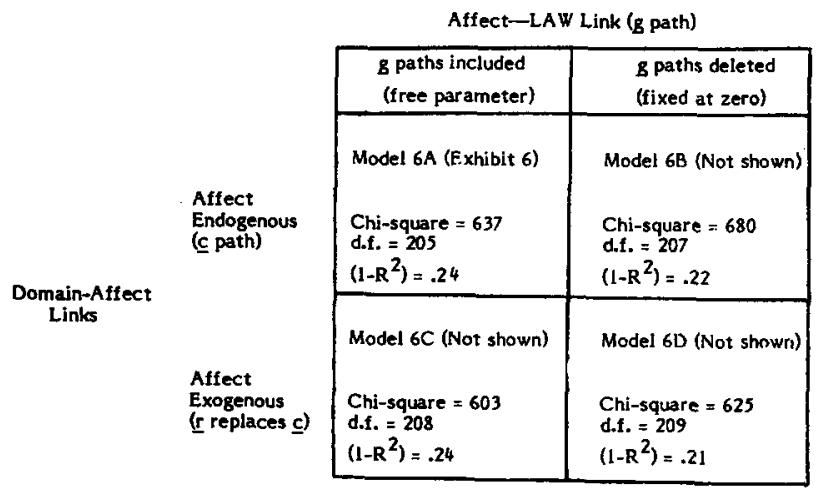

Exhibit 7. Measures of fit (chi-squares, degrees of freedom, and variance left unexplained in the LAW factor) for the model of Exhibit 6 and three variants. 
LAW path coefficients $(g)$ turned out to be small $(0.18$ and 0.16$)$. An alternative model, which we shall call ' $6 \mathrm{~B}$ ', was therefore run to test the effect of deleting the $g$ paths by fixing their values at zero. While the increase in chisquare more than reflected the increase in the degrees of freedom due to the delection of the $g$ paths, we put greater weight in this comparison on the external validity of the models as reflected in the residual coefficient $\left(1-R^{2}\right)$ for the prediction of LAW. There was little difference between the models in this respect. Thus while Model 6B indicated that any direct effect on LAW must be very small, the comparison with $6 \mathrm{~A}$ showed that affect did not in fact make any significant contribution to the prediction of LAW over and above that already contained in the domain affects. ${ }^{9}$

Models $6 \mathrm{C}$ and $6 \mathrm{D}$ are variants of $6 \mathrm{~A}$ and $6 \mathrm{~B}$, respectively, and differ from them only in that the affect factors are made exogenous - i.e., the coefficients ( $c$ paths) for the regression of affect on the domains are replaced by correlation coefficients $(r$ 's). As far as the $g$ path is concerned, comparison of the former two models replicates the comparison of the latter and leads to the same conclusion, namely that affect makes no direct contribution to the LAW factor. However, it is the comparison between the $c$ paths and the $r$ 's that now concerns us. One of the consequences of making affect an exogenous variable can be studied by comparing the set of domain-affect correlations from Model $6 \mathrm{C}$ with the $c$ path coefficients from Model 6A. (The $c$ path coefficients for Model 6B were virtually identical.) This is done in Exhibit 8.

It will be recalled that the domain factors included in these models were selected because of the range of covariation that their constituent items showed with affect. ${ }^{10}$ The correlations in the top half of Exhibit 8 reflect this range. There is not only the difference in overall level as between domain

\begin{tabular}{|c|c|c|c|c|c|}
\hline & \multicolumn{5}{|c|}{ Dornain Factors } \\
\hline & $D_{1}$ & $\mathrm{D}_{2}$ & $\mathrm{D}_{3}$ & $D_{4}$ & $\mathrm{D}_{5}$ \\
\hline \multicolumn{6}{|c|}{ Correlations (from Model 6C) } \\
\hline Positive Affect & .48 & .40 & .20 & .22 & .14 \\
\hline Negative Affect & -.37 & -.37 & -.21 & -.29 & -.36 \\
\hline \multicolumn{6}{|c|}{$\frac{\text { Regression Coefficients }}{\text { (trom Model 6A) }}$} \\
\hline Positive Affect & .41 & .19 & .12 & .01 & .17 \\
\hline Negative Affect & -.15 & -.12 & -.09 & -.04 & -.18 \\
\hline
\end{tabular}

Exhibit 8. Links between domain factors and affect. 
factors 1 and 2 versus 3 and 4, but also interesting discrepancies between the connections with the two affects. The family domain, for example, is more strongly linked to positive than to negative affect, while the reverse applies to the relaxation domain. This substantively interesting pattern of association disappears when we turn to the regression coefficients in the lower half of Exhibit 8 . Three of these are near zero, while most of the rest tend to be very small. These results lead us to believe that the original correlations are a better guide to the relationship between the domains and the affect factors than are the path coefficients, and constitute one reason for preferring models in which affect is made an exogenous variable.

Consistent with this preference is the finding that models where affect is made exogenous provide a better fit to the observed data than models where it is endogenous. The latter models, represented by $6 \mathrm{~A}$ and $6 \mathrm{~B}$ in Exhibit 7, have larger chi-squares yet smaller degrees of freedom than the comparable exogenous models, $6 \mathrm{C}$ and $6 \mathrm{D}$, respectively. A decrement in degrees of freedom means that more parameters have been estimated, and it is usual to find that chi-square decreases, indicating some degree of improvement in fit. It is then a question of whether the saving in chi-square points is sufficiently greater than the loss of degrees of freedom for the improvement in fit to be judged substantial. In this case however chi-square has increased, so that it is quite clear that the fit is worse.

The reasons for the poorer fit of the endogenous models are instructive. Although the path coefficients between the two affect factors were fixed at zero, the estimated correlation between them turned out to be 0.13 in $6 \mathrm{~A}$ and 0.16 in Model 6B. The original correlation between the affect scales was close to zero, so a large residual was introduced at this point. The spurious correlation between the affect factors arises, it would seem, because when affect is made endogenous the models assume that both affect factors are joint effects of the same causes, namely the domain factors. Here then is a further reason for thinking that the assumption of a cause-effect relationship between domains and affect is erroneous.

We may now review the basic assumptions in the original model schematised in Exhibit 5. Affect was placed in an intervening position in this model because initially it seemed plausible to consider the domain evaluations as a set of causes acting together to produce an overall affective response which in turn was incorporated into the assessment of global well-being. (It did not and does not seem plausible to assume that global affect could be antecedent 
to and act as a cause of the specific domain evaluations.) However, now that the consequences of assuming affect to occupy an intervening position have been made explicit in terms of structural equations, we find this model to be untenable. We were led at this point to a model in which affect is made exogenous alongside the domain evaluations, and in which affect has no direct effect on the assessment of life-as-a-whole. This model has its shortcomings, as will be seen, but compared to the intervening position model it provides a more adequate as well as a more parsimonious account of the observed data.

Domain-specific affects. A model in which affects and domains are both exogenous offers no hypotheses about either the nature of the associations between the domains and affect or the reasons why affect should have no direct effect on the assessment of life-as-a-whole. These two shortcomings of the model (there is a third, much more important, to which we shall come presently) can be met, at least theoretically, if we postulate that each domain item (or domain factor) has associated with it a specific element of affect. A model that incorporates this assumption may be schematised as in Exhibit 9.

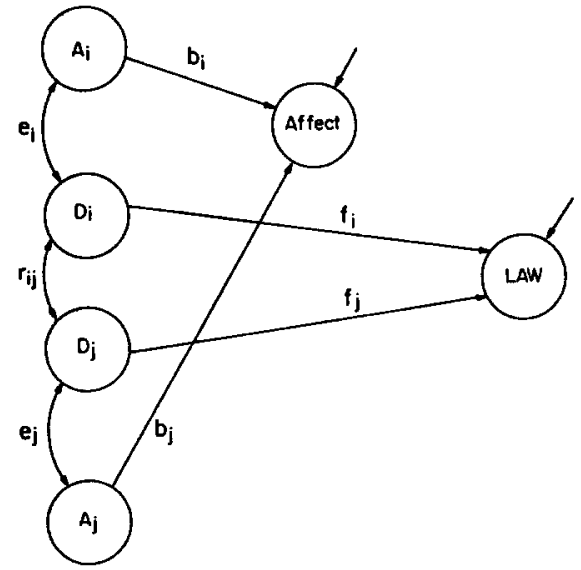

Exhibit 9. Basic form for path model with global affect explained by domain-specific affects, and with life-as-a-whole (LAW) explained by domains.

In Exhibit $9, A i$ and $A_{j}$ are specific elements of affect associated with domain factors $D_{i}$ and $D_{j}$. The contribution of the domains to global affect can now be interpreted as the joint result of the direct effects $b_{i}, b_{j}$, of these domain-specific affects without needing to postulate direct effects from the domain evaluations (the troublesome $c$ paths in Exhibit 5); the correlation 
between a domain and global affect receives an interpretation as a coordinating-path effect given by the product $e_{i} b_{i}$; and the absence of a direct path between global affect and LAW can be explained by assuming that the affect is already in the domains. Hence the contribution of affect to life-as-a-whole is accounted for by the domain-specific affects.

However plausible, the model in Exhibit 9 must remain untested as we do not have measures of domain-specific affects in any currently available data set. Moreover, a major theoretical shortcoming can be seen if we ask what else, other than affect, is transmitted along the $f$ paths between domain evaluations and LAW. We know that more than affect is transmitted since the domains account for much more variance in LAW than does affect. Our theory, of course, says that the missing factor is cognition. Section 3 showed how factor models which included the cognitive factor could be constructed from the available data. The role of cognition in the judgement process can be examined by running the path analogues of these factor models. As will be seen in the next section, the models that ensue lead to a view of the underlying dynamics of the judgement process that is very different from that of the affect-only model in Exhibit 9.

\subsection{Affect-Cognition Models}

The basic characteristics of the model to be considered in this section may be schematised as in Exhibit 10.

Following the work in Section 4.2, affect in Exhibit 10 (again shown as a

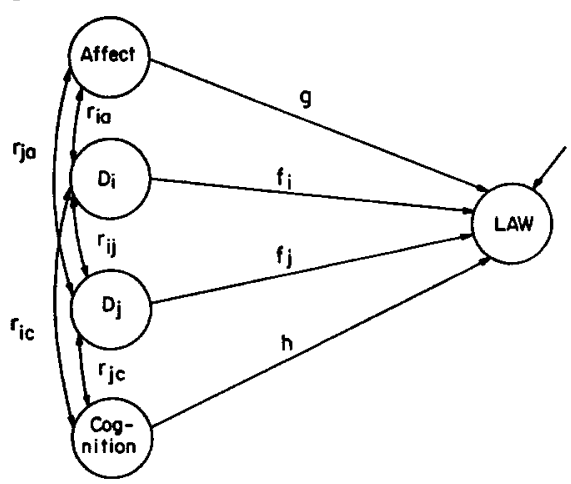

Exhibit 10. Basic form for path model life-as-a-whole (LAW) explained by affect, cognition, and/or domains. 
single variable for simplicity) is portrayed as exogenous and linked to the domains $D_{i}$ and $D_{i}$ by the correlations $r_{i a}$ and $r_{j a}$. A cognitive factor has been introduced and is also treated as exogenous, being linked to the domains by correlations $r_{i c}$ and $r_{i c}$. The path from cognition to the LAW factor is denoted by $h$. The paths from the domains are denoted by $f_{i}, f_{j}$, and the path from affect by $g$ as previously (Exhibit 5).

If the paths $f_{i}$ and $f_{j}$ from the domains are set equal to zero in the model in Exhibit 10, then the input to LAW would consist of two parts - affect and cognition. The paths $g$ and $h$ would be unaffected by the domain evaluations, so that the relations between affect, cognition, and LAW could be modelled without reference to the domains.

To anticipate, the discovery that paths $f_{i}$ and $f_{j}$ really would be zero in this model was the main outcome of the attempt to implement the model of Exhibit 10.

Cognition as residual variance in the $L A W$ factor. It was not immediately obvious, but on reflection it was realised that the lack of a direct effect of domains on the LAW factor ( $f$ paths equal to zero) is implicit in the definition of the cognitive factor. The nature of the implication can be clarified by considering the results for a path model that includes cognition, affect, and LAW, but excludes the domain evaluations. Exhibit 11 shows the results of estimating the parameters of such a model using the LISREL program. ${ }^{11}$

The validity coefficients for the affect measures and the loadings of the

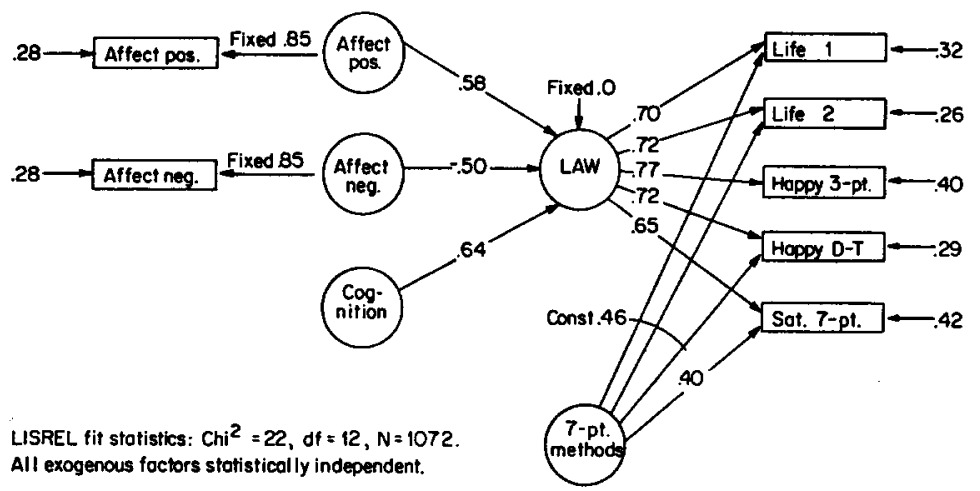

Exhibit 11. Factor-path model with life-as-a-whole (LAW) explained by affect and cognition, and with allowance for random and correlated measurement errors. 
global measures on the method factor in Exhibit 11 are a little different from the values subsequently found to be optimum for these coefficients (see Andrews and McKennell, 1978). Otherwise the results in Exhibit 11 serve very well to bring out the salient theoretical issues raised by models of this general type.

Exhibit 11 portrays a causal structure relating unobserved variables in which the LAW factor is the endogenous variable being predicted. But no residual coefficient is allowed for the LAW factor. The cognition factor is really acting here as a residual input. This accords with the operational definition of cognition that we have had to follow in order to proceed with the analysis: Cognition is the factor responsible for that part of the variation in measures of global well-being which remains after the contributions of affect and method components have been removed. In non-causal factor models, such as those in Exhibits $2 a$ and 3a, cognition could be factored directly out of the observed variables, and its contribution to these variables determined, because the causal relationship between the factors were not under study. For the causal influence of cognition on the LAW factor to be studied, however, cognition must be defined independently of both the LAW factor and the variables used to define this factor. The strategem of obtaining cognition by residualising out of the LAW factor seems reasonable enough if we can accept, first, that the model has succeeded, as intended, in stripping away random and correlated errors in the measurement of the LAW factor and, second, that the variance then unaccounted for by affect must be ascribed to cognition.

The most important outcome of estimating the model of Exhibit 11 is the very substantial $g$ paths for the inputs from affect to the LAW factor. These contrast with the very small $g$ paths described in Section 4.2 for models without cognition (Exhibits 6 and 10) and will be interpreted shortly.

Cognition as residual variance in the $L A W$ indicators. Because we have a set of six global indicators an alternative strategem is to obtain the cognitive factor by residualising affect out of some of these indicators while using the remaining ones to define the LAW factor as before. However, residualising cognition out of the indicators of LAW is not so very different from residualising it out of the LAW factor itself, and cannot be expected to alter the main outcome. The alternative strategem was in fact adopted for the model in Exhibit 12. (We may note that the model in Exhibit 12 and a variant derived later are both path analogues of the model in Exhibit 4, that a cognitive factor was also defined in the models in Exhibit $2 a$ and $3 a$, and that for all of 
these models the loadings of the global indicators on the cognitive factor vary very little, and hence give this factor a constant meaning throughout.)

In the model of Exhibit 12 it can be seen that the $g$ paths from positive and negative affect are again substantial. We were also concerned to examine the role of the domain evaluations along with cognition and affect in the total causal structure. ${ }^{12}$ The paths from the domain factors to LAW were left as

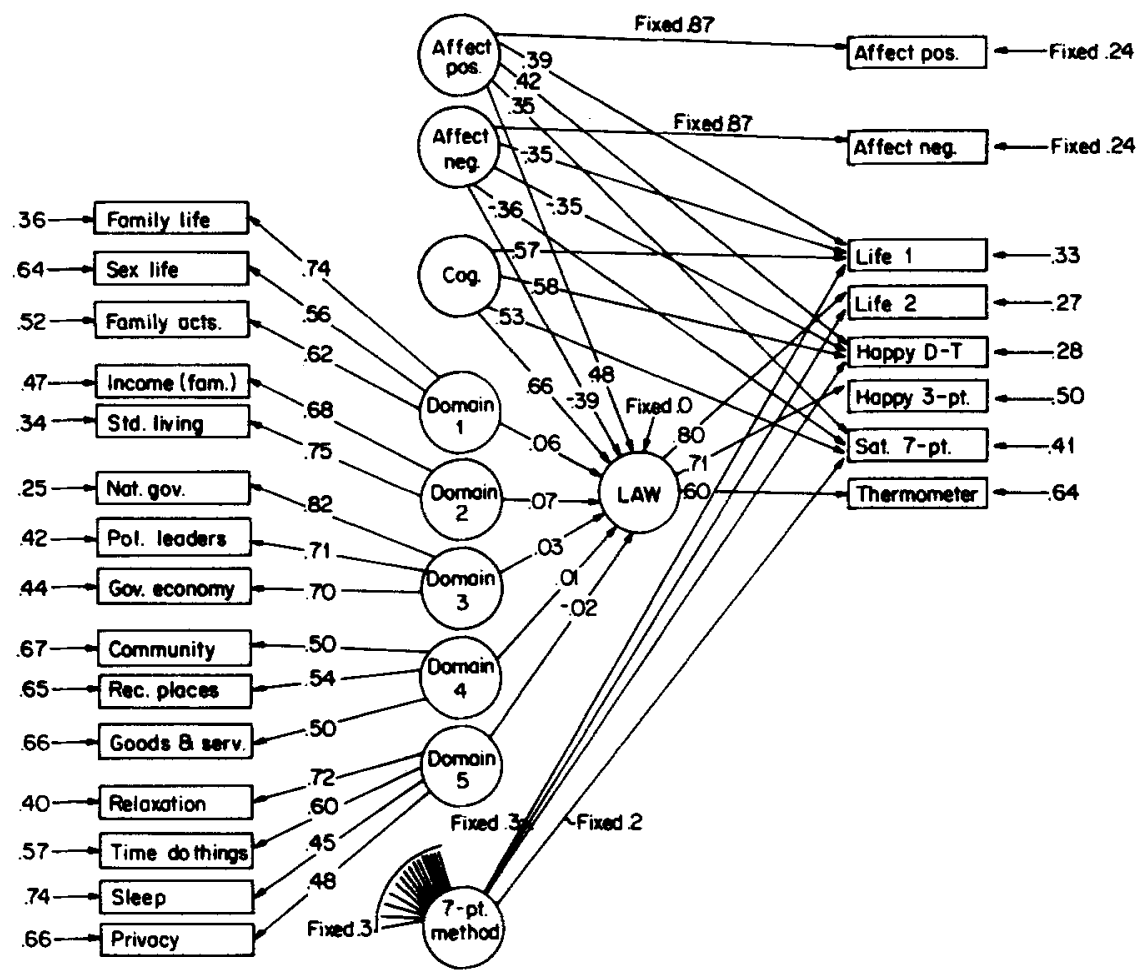

LISREL fit statistics: $\mathrm{Chi}^{2}=591$, of $=196, \mathrm{~N}=1072$

Estimated relationships ( $r$ 's) among exogenous foctors [ $f=$ fixed]

\begin{tabular}{|c|c|c|c|c|c|c|c|c|}
\hline & $+A$ & $-A$ & $\cos$ & 01 & $\mathrm{D} 2$ & D3 & 04 & D5 \\
\hline $\begin{array}{l}\text { Affect neg. } \\
\text { Cognition } \\
\text { Domain } 1 \\
\text { Domain } 2 \\
\text { Domain } 3 \\
\text { Domain } 4 \\
\text { Domain } 5 \\
7 \text {-pt. mid. }\end{array}$ & $\begin{array}{l}1.00 \\
1.00 \\
.48 \\
.40 \\
.20 \\
.22 \\
.19 \\
1.00\end{array}$ & $\begin{array}{r}f .00 \\
-.38 \\
-.37 \\
-.21 \\
-.30 \\
-.36 \\
t .00\end{array}$ & $\begin{array}{r}.51 \\
.43 \\
.04 \\
.20 \\
.34 \\
.00\end{array}$ & $\begin{array}{r}.56 \\
.11 \\
.29 \\
.49 \\
.00\end{array}$ & $\begin{array}{r}.29 \\
.57 \\
.51 \\
.00\end{array}$ & $\begin{array}{r}.39 \\
.22 \\
f .00\end{array}$ & $\begin{array}{r}.50 \\
1.00\end{array}$ & 1.00 \\
\hline
\end{tabular}

Exhibit 12. Factor-path model with life-as-a-whole (LAW) explained by affect, cognition, and/or domains, and with allowance for random and correlated measurement errors. 
free parameters to be estimated by the model. As can be seen in Exhibit 12 the estimates for these path coefficients approximated zero in all cases. We therefore ran the model again with these paths fixed at zero. The results were very similar. Since the new model (not shown) had five fewer paths, its degrees of freedom were five points higher, and since its chi-square value increased by only about the same amount, there was a clear indication that the parameters linking the domains to the LAW factor in the model of Exhibit 12 did not produce any real statistical improvement in the fit of the model. The true value of these parameters in this model, equivalent to the $f$ paths in Exhibit 10 , can therefore be taken as zero.

The value for the paths linking positive affect, negative affect, and cognition to the LAW factor in our revised model were, respectively, 0.54, 0.44 and 0.72 , - only slightly different from the values for the model in Exhibit 12 . It is notable, too, that these values are very near those obtained for the model of Exhibit 11, which did not include the domains. (The minor differences are most probably due to the differences in the method effects.) We see again, therefore, that the domain assessments can even be excluded from the causal model without altering the estimates for the direct effects of cognition and affect on the LAW factor.

We conclude that in models that incorporate both affect and cognition factors, and that define cognition as a residual, the flow of influences determining the LAW assessments will be by way of the cognitive and affective factors.

Domain-specific cognitions. As with the affect-only model, the correlations between the domains and affect remain unanalysed in the affect-cognition model. But the affect-cognition model has a cognition factor as well as affect in the exogenous position. The theory of domain-specific affects, presented in Section 4.2 , now needs to be extended if an interpretation is to be provided for the domain-cognition correlations as well as the domain-affect correlations. We therefore postulate the existence of domain-specific cognitions. A model that incorporates the assumption of domain-specific affects and domain-specific cognitions may be schematised as in Exhibit 13.

In Exhibit $13, A_{i}$ and $A_{j}$ are specific elements of affect, and $C_{i}$ and $C_{j}$ are specific elements of cognition associated with, respectively, domain factors $D_{i}$ and $D_{j}$. The correlation between a domain $D_{i}$ and global affect receives an interpretation as a coordinating path effect given by the product $e_{i} b_{i}$; and the correlation between a domain and global cognition as the coordinating path $k_{i} l_{i}$. 


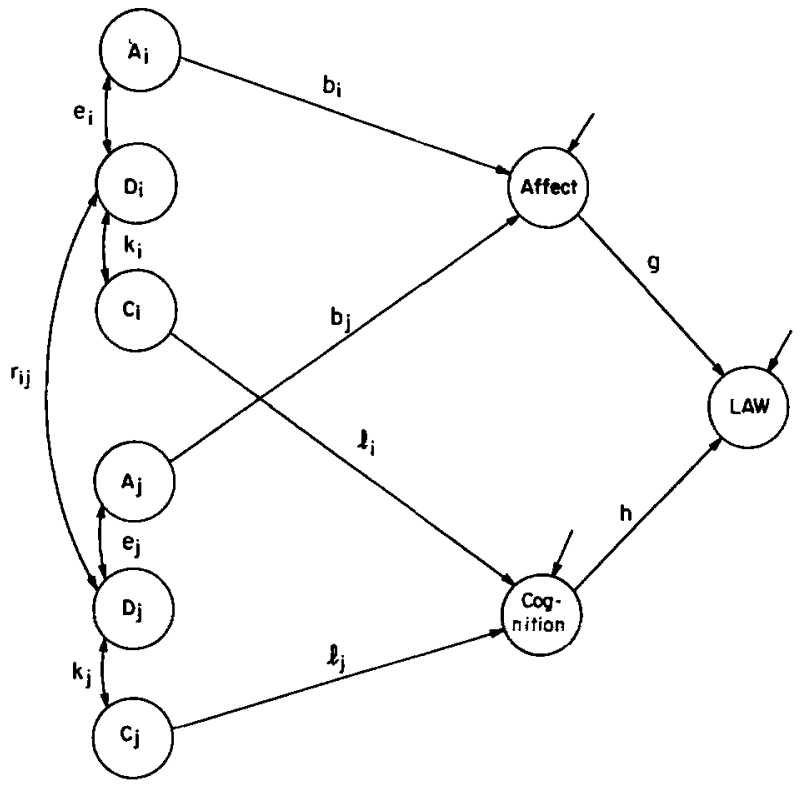

Exhibit 13. Basic form for path model with exogenous domain-specific affects and cognitions, and with global affect and global cognition intervening between domains and life-as-a-whole (LAW).

\subsection{Evaluation of Affect-LAW versus Affect-Cognition Models}

According to whether we adopt the affect-LAW or affect-cognition model as an explanation for well-being evaluations, we arrive at very different conclusions about the role played by affect and by domain evaluations. Comparisons of these models will be facilitated if the main outcomes of the detailed discussions in Sections 4.2 and 4.3 are presented in schematic form as in Exhibits $14 \mathrm{a}$ and $14 \mathrm{~b}$.

The schema show the domain factors $D_{i}$ and $D_{j}$ along with by-now familiar concepts of the study, affect, cognition, and life-as-a-whole (LAW). The ovals round each variable are intended to convey that it is the relationship between factors or unobserved variables that are under study - random and correlated errors (methods effects) have been allowed for and so do not enter into the comparison. Exhibits 14a and 14b may be regarded as schema for Model 6D (Exhibit 7) and the model in Exhibit 12, respectively, which show in detail how the allowance was made. 


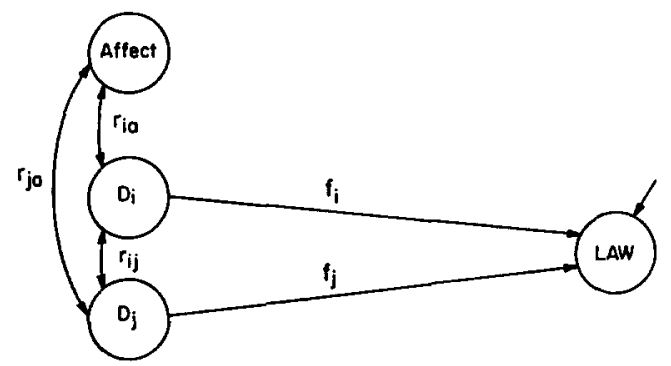

Exhibit 14a. Basic form for path model with life-as-a-whole (LAW) explained by domains and with affect exogenous - the 'Affect-LAW' model.

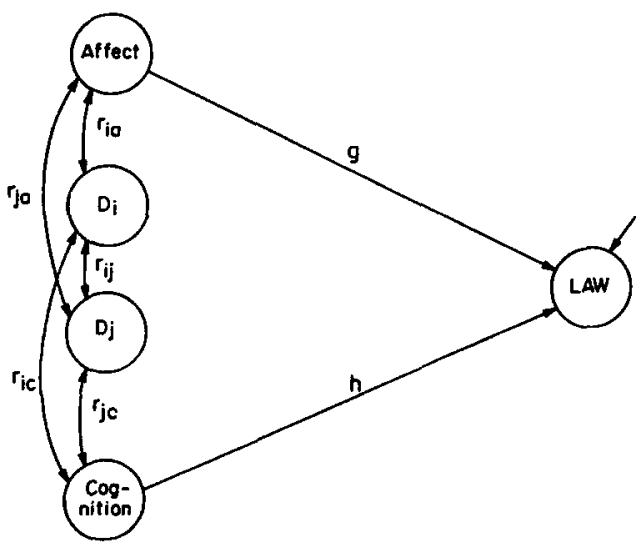

Exhibit 14b. Basic form for path model with life-as-a-whole (LAW) explained by affect and cognition, and with domains exogenous - the Affect-Cognition model.

It is possible to argue, on only statistical grounds, that the affect-cognition model is preferable in that that introduction of a cognitive factor does provide a marginally better fit to the observed data (as demonstrated in Section 3). However, the substantive implications of the paths in the models provide even more compelling reasons for preferring the affect-cognition model.

There are at least two such implications. First, there is the absent $g$ path in Exhibit 14a. The implication here is that respondents asked to evaluate their life-as-a-whole do not take into account their, as it were, 'gut feelings' about 
the emotional quality of their life. This is intuitively difficult to accept. The substantial $g$ path in the affect-cognition model, on the other hand, suggests that respondents' sense of the emotional quality of their life is a factor that has a direct impact, whether it be conscious or unconscious, on evaluations of life-as-a-whole, even if it is not the only factor.

A second source of difficulty with the affect-only model relates to the implication that the $f$ paths have for a cognitive factor. The model requires that the influence of affect is transmitted along the $f$ paths. But clearly more than affect is being transmitted because the domains account for much variation in LAW beyond the contribution of affect. If we ask what else is being transmitted, we are led back to the postulation of cognition. It did not prove possible to integrate a cognitive factor into the affect-only model without arriving at the quite different affect-cognition model in which the $f$ paths turned out to be zero. It is not a case of one model being a development out of the other. The two seem incompatible.

If the above theory about the existence and role of a cognitive factor is correct, therefore, the affect-cognition model seems preferable to the affectonly mcdel. However, whether people do in fact form evaluation of life-as-awhole by 'combining' some sense of global affective evaluation with some global cognitive evaluation (as the affect-cognition model postulates), or whether some other mechanism that might more directly involve combining domain evaluations (which might themselves already incorporate certain domain-specific affects and cognitions) is used, is not something that can be definitely determined with the available data. Our modelling analyses are helpful in showing the implications of different theoretical approaches and have suggested the desirability of obtaining certain further types of data in the future (i.e., measures of domain-specific affects, domain-specific cognitions, and global cognition). But, as in any modelling activity, the results we have obtained are dependent on the particular models that have been examined. The absence of observed measures of global cognition and the consequent necessity for us to define it as a residual, and the absence of any data on affects or cognitions linked to specific life concerns and hence the impossibility of including these in our models, may well have influenced the results we have obtained. 


\section{OTHER DATA SETS}

\subsection{British Data}

A survey of perceived well-being in Britain by Abrams and Hall (Abrams, 1976; Hall, 1976) utilised the Bradburn affect scales and a range of domain evaluations together with a large and varied set of global indicators of life quality. From the perspective of the present paper it is important to note that the affect-cognition model fitted the British data very much better than did the affect-LAW model, thereby replicating the essential result obtained with the American data, as described in Section 3.

Hall (1975) has reported a path analysis of the British data in which the affect scales were introduced as intervening variables as in Exhibit 5 above. Six domain-evaluation measures were employed in this analysis: Family, Living standard, Health, Financial situation, Job, and Leisure. An almost identical set of six domains, along with Bradburn's affect scales and a global measure of life-as-a-whole, could be selected from one of the data sets in the Andrews-Withey series - their November 1972 Form 2 national survey (Andrews and Withey, 1976). It proved possible therefore to replicate Hall's analysis exactly with these American data.

There was an impressive similarity in the main structural features of the results. First, the direct effects of the affect measures on the global evaluations were equally small. The path coefficients (the $g$ paths in Exhibit 5) for positive and negative affect, respectively, were 0.13 and 0.12 in the British data and 0.10 and 0.11 in the American data. ${ }^{13}$ The variance explained in the measure of global well-being was high, with an $R^{2}$ of near 0.50 in both analyses but the direct contribution of the affect paths to this prediction was negligible, as implied by their miniscule path coefficients. (See also McKennell, 1978, Table IV.) This is not so elaborate an analysis as the one pursued with the American data in Section 3, since no allowance was made for random or correlated errors in the observed variables, ${ }^{14}$ but it is adequate to show that the first main finding regarding the intervening variable model for affect demonstrated in Section 4.2 holds for the British data just as it does for the American: The path from affect to the global measure, the $g$ path in Exhibit 5 , is small and can be eliminated without loss of explanatory power.

The second main finding in Section 4.2 was that the paths from the domain evaluations to the affect factors, the $c$ paths in Exhibit 5 , need to be 
replaced by correlational links. This result emerges even more conclusively when Hall's model is replicated with the American data. The variance in each affect measure explained by the set of domains is small $\left(R^{2}\right.$ less than 0.20$)$ in both the British and American data sets. However, the most important finding is that the coefficients for most of the paths from domains to affect (the $c$ paths in Exhibit 5) were less than 0.10. Yet in both sets of data the domain-affect correlations were mostly subsantial, at or above 0.20 . It is difficult to believe that activities in such areas as family, leisure, and job generate no affect or hardly any affect. It is easier to believe that the original correlations are a better guide to the relationships between the domains and affect than are the path coefficients. We are thus led, as with the discussions of Exhibit 8 in Section 4.2, to a preference for a model in which affect is made an exogenous variable. We thus replicate with British data, albeit at a simplified level, the two principal conclusions drawn from the more elaborate analyses of American data.

In Section 4.2 a further theoretical development was presented which sought to explain the absence of $c$ and $g$ paths by positing that each domain evaluation was associated with a domain-specific element of affect. Although this theory cannot be tested directly, since we do not have domain-specific affect measures, some indirect evidence is forthcoming from running the regressions implied by Hall's model, and its Andrews-Withey replication, in stepwise fashion, adding one domain at a time. For the prediction of global well-being (LAW), the domains were allowed to enter only after first forcing in the affect measures. The two affect measures being uncorrelated, the values of $g$ before any domains were added were the original correlation of each affect scale with the global measure: 0.25 for positive affect and 0.37 for negative affect in the Abrams-Hall data ( 0.36 and 0.32 , respectively, in the Andrews-Withey data). The value of $g$ then fell rapidly with the first domains that entered the stepwise regression and then more slowly as further domains are added until there was no decrement detectable (in the second decimal place) for the last few domains. So similar were the results that the curve for the decline of $g$ as domains were added in the Abrams-Hall data could be almost exactly superimposed on the plot for the Andrews-Withey data. ${ }^{15}$ The behaviour of the $\boldsymbol{g}$ path here as domains were added is consistent with the theory that affect adds little to the power of the domains to predict life-as-awhole because the elements of affect are already contained in the domain evaluations. As domains are added, it would seem that affect elements are 
added also, until the point where the entire contribution of global affect is accounted for.

\subsection{NORC's American Data}

The above analyses of the data from British and American surveys was especially valuable because of the near identity that could be secured in the set of variables examined. Burt $e t$ al. (1978) have published analyses of a correlation matrix for NORC data between ten domain satisfaction measures, Bradburn's affect scales, and two global measures of well-being. Unfortunately, the set of domains overlaps only partially with the sets just discussed, and is much lower in predictive power, ${ }^{16}$ no doubt in part because the important Family domain is not included. Nevertheless, when the stepwise regression just described was repeated with the NORC data, the same two basic structural characteristics of the result emerged: (1) The affect measures did not add to the predictive power of the domains in explaining the variance in the global indicators. In stepwise regression most of the variance in global satisfaction was accounted for by the first two domains to enter, and the values of the $g$ coefficients rapidly fell to a very low value $(0.11)$ as further domains were added. (2) Only three domains had Beta coefficients ( $c$ paths in Figure 5) greater than 0.10 in the prediction of affect $(0.13,0.17$, and 0.22$)$. Despite some apparent substantive differences, therefore, the NORC data set again replicates the basic structural characteristics noted in the other surveys of perceived quality of life. ${ }^{17}$

\section{SUMMARY AND DISCUSSION}

Our starting point for this program of secondary analysis of data from quality of life surveys lay in the observation that ratings of happiness and life satisfaction often correlate differently with outside variables such as age, income, and education. A special analysis of data from the cross-tabulation of happiness against satisfaction ratings supported the interpretation that the former were more affective and the latter more cognitive. At the same time, the analysis also suggested that these two types of measures, in common with other currently-used rating-scale indicators of perceived well-being, were compounds of both cognition and affect, and differed only marginally in the extent to which one or the other of these factors was being tapped. A 
measure of affect was available in the scales developed by Bradburn (1969), but there was no measure of cognition. We therefore conceived the idea of operationally defining cognition in terms of the variance that remained in global indicators of well-being after that due to affect and two types of measurement error had been partially out. The initial ideas were set out in a paper by McKennell (1978). The present paper develops a series of structural equation models in which the logical consequences of the assumptions underlying these ideas are made explicit.

In Section 3, models containing a cognitive factor were compared with models that did not. This comparison was made for models of increasing complexity. Beginning with models that contained only global indicators plus factors for affect and life-as-a-whole, we proceeded to models in which domain evaluations were added, and finally to models which also added a cognition factor. Through this series of models, the loadings on the cognition factor and the loadings on the other factors changed little. Despite its status as a residual factor, defined in terms of its structural relations with other variables rather than through its own indicators, the cognition factor retained a consistent meaning across the separate models. Moreover, in all the major comparisons, models that contained the cognition factor fitted the data better than models that did not.

Thus encouraged, we moved on from the static picture of the factor structure of well-being, in Section 3, to consider more dynamic models, in Section 4 , that sought to portray the flow of determining influences between factors.

It is worth looking back at this point to the notions about the dynamics of the judgement process that prevailed, and which we shared with others, at the time we came into the present investigation. Previous investigators (notably Andrews and Withey, 1976; Campbell et al., 1976) had discussed the way the LAW factor or its indicators can be predicted by a linear summation of separate domain evaluations. For the most part, the emphasis in these discussions was on statistically accounting for the variance in global well-being measures. Andrews and Withey (1976, pp 108-109), however, briefly suggested that

the statistical model can also be considered a psychological model. Not only is the model that method of combining feelings that provided the best predictions, it is also our best indication of what may go on in the minds of the respondents when they themselves combine feelings about specific life concerns to arrive at global evaluations. Thus, our statistical model can also be considered as a simulation of psychological processes. 
A model was postulated in which domain evaluation factors were portrayed as direct dertermining influences for life-as-a-whole assessments (see Andrews and Withey 1976, Exhibit 6.4).

Although the precise sense in which this model simulated psychological processes was not spelled out, the model might be justified as a representation of the unconscious workings of the schemata in the minds of respondents. However, the attempt in Section 4.2 of the present paper to integrate global affect into the domain-LAW model led to consequences which were intuitively difficult to accept. On the other hand, the attempt in Section 4.3 to integrate both global affect and global cognition led to a more appealing model, but one in which the assumption that the domains have a direct causal impact on LAW assessment had to be abandoned.

To recapitulate briefly, the affect-LAW model considered in Section 4.2 kept the original feature in which the domains were assumed to have a direct causal impact on the LAW assessments, while attempting to explain the correlation that occurs between affect and LAW. One possible interpretation of this correlation would be that, when asked to assess their feelings of global well-being, respondents actively and consciously take into account the emotional quality of their lives. If this had been the case, however, the model should have shown a direct effect in terms of causal paths running from the affect factors to the LAW factor. In running the model, however, we found that if such paths were included in this model, their influences were so small that they made no significant contribution to the explanation of variance in the LAW factor. The affect variables, in other words, added nothing to the variance in the LAW factor that was not already accounted for by the domain evaluations.

Moreover, we found clear evidence that a model which placed global affect as an intervening variable between the domain factors and the LAW factor yielded a poorer fit to the data than did a model which positioned global affect as an exogenous variable - correlated with, but not directly influenced by, the domain assessments.

It is possible to interpret the role of affect here by saying that its influence is somehow entailed in the action of the domains, and to this explanation we return shortly. A main conclusion from our analysis of the affect-LAW model is that in this model there is no direct path between affect and LAW. It is this consequence of the affect-LAW model that is intuitively difficult to accept. Since the initial domain-LAW model, which assumes that domain evaluations 
are direct causes of LAW assessments, leads to this consequence, it too is put in doubt.

Section 4.3 attempted to integrate both a cognitive factor as well as affect into the basic domain-LAW model. The consequences of this attempt was a model, the affect-cognition model, which was incompatible either with the basic domain-LAW model or its extension into the affect-LAW model. For in the affect-cognition model we found the paths denoting a direct impact between domain evaluations and LAW become redundant. All the non-error variance in LAW could be explained as the joint effects of cognition and affect. While this was a direct result of the way cognition had been defined (i.e., as a residual), the effect - surprising at first - was that in this model the correlation between the domain factors and LAW came about indirectly through the correlation the domains had with cognition and affect.

If this affect-cognition model were to be accepted, then the basic domainLAW model would have to be rejected. On intuitive and substantive grounds there are some appealing aspects to the affect-cognition model. The path between affect and LAW is restored, and this seems intuitively reasonable. But now there is a second path from cognition. The model states that in arriving at global assessments of life quality respondents weigh directly both affective and cognitive considerations, so that the final assessment is a compound of the two. This accords with our original theorising. As far as this model is concerned, global assessments can be obtained quite separately from the domain evaluations. This accords with the facts. If we ask for domain evaluations respondents can give them, and if we call for global assessments, we can get these also. It seems that these are distinct approaches to quality of life that may not be consciously connected by our respondents at the time they respond to the survey questions. The connection is made theoretically and statistically by us as investigators. As far as our respondents are concerned, there is an important sense in which the global assessments are domain-free, and the affect-cognition model incorporates this feature.

The linear additive relation that exists between domain evaluations and global assessments of life quality has been seen by previous investigators as a major piece of evidence for the construct validity of their measures. Nothing written here changes that verdict. But the status of the relationship requires further explication. The hypothesis was suggested (see Exhibit 13) that associated with each domain is a domain-specific element of affect and, similarly, a domain-specific element of cognition. Thus, by summing the domains one 
would sum the elements of affect and the elements of cognition until the contribution of global affect and global cognition to the global assessment of well-being would be accounted for statistically. This hypothesis is consistent with the position that it is the global affect and global cognition and not the domain evaluations that have the direct impact on the final LAW assessment - i.e., this hypothesis says that the contribution of the domains is indirect via their associations with global affect and global cognition.

The associations between the domains and global affect and global cognition also need interpreting in their turn. The hypothesised model (Exhibit 13) was constructed so that there are no direct paths between the domains and either global affect or cognition. The association here is also indirect via the domain-specific elements of affect and cognition. This is in line with what we know about respondents' ability to make global assessments of affect without apparently making immediate reference to evaluations of particular domains. The essence of Bradburn's achievement in developing his affect scales was to have operationalised the concept of global affect by means of domain-free measures. Whether or not the concept of global cognition can be operationalised in similar terms remains to be seen.

We know little about the cognitive factor other than the way it operates in the models we have constructed. Certainly we would like to know more. But these models do provide some construct validity. The definition of a factor in terms of the residual variance it can account for is a common operation in conventional factor analysis of the exploratory kind. The confirmatory mode of factor analysis followed in this paper provides a more direct route to construct validity. Factor axes are positioned not through arbitrary rotation procedures but in terms of the identification constraints imposed by a theoretical model. Moreover, in second-order confirmatory factor analysis (producing what we have termed factor-path models) the factors are put to use in models which seek to explain the relationship between them. The relative value of a particular model in relation to alternatives is then assessed in terms of both goodness of fit and substantive interpretation. There is every reason to suppose that the interpretation that can be placed on factors in models that survive this process will have construct validity.

An interpretation that can be placed on the affect-cognition model is that the causal pathways in it might represent the respondents' mental processes during the interview situation, whereas the correlational links between variables left exogenous in the model might be set up in the respondents' minds by 
their previous life experiences outside the interview situation. Thus, this model would suggest that respondents actively integrate global affect and global cognition in arriving at their global assessment of live quality. However, the linkages that assessments of life-as-a-whole have with the domain evaluations would depend on the associations domains have with global affect and global cognition by way of the domain-specific affects and cognitions, linkages which stem from an individual's living experiences prior to the interview. Thus this model is quite consistent with the finding that a linear additive combination of the domain assessments can be used to predict LAW assessments, but it posits very different account of the psychological processes entailed in the connection.

It is a task for future research to attempt to construct direct indicators for global cognition which will be domain-free and analogous to the scales that Bradburn has devised for the measurement of affect. It is too early to say what these indicators will look like. As was discussed by McKennell (1978), the way forward may be through the study of different types of comparison criteria - values, standards, aspirations, goals - in the assessment of global well-being. A beginning has been made here in the work of Abrams (1975), Hall (1975), Mason and Faulkenberry (1978) and Bortner and Hutsch (1973). So far no generalised measure corresponding to the cognition factor has been devised, though this has not been among the objectives of the investigators. Andrews and Withey (1976) proposed a Domain-by-Criteria Model which so far has been little researched. The criteria in their list were quite general (for example, amount of challenge, privacy, comfort, fun, variety and diversity, independence and freedom, responsibility, and so forth). The model assumed that people evaluated the criteria in relation to the domains (or vice versa), but both the criteria and domains could also be investigated independently. Further study of the framework of criteria in the context of such a model might throw further light on the cognitive factor. Bradburn's research showed, surprisingly, that the measurement of affect required two factors. There was no problem in incorporating this two dimensional concept in our models. For convenience we have treated global cognition as a single factor. But we would not be surprised if several factors proved necessary to cover the concept, nor would we anticipate any difficulty in principle in exetending our models to cover multiple dimensions of cognition.

At the present stage of research, the notions of domain-specific affect and domain-specific cognition remain purely hypothetical. But it should prove 
possible to operationalise these concepts so that the models in which they appear can be tested empirically. With respect to domain-specific cognition, the most illuminating study that we have up to now of the working of comparison standards was made within the specific domain of housing satisfaction (Campbell et al., 1976, Chapter 6). These investigators have recently embarked on a survey in which comparison standards in several other domains are being subjected to similar intensive study. ${ }^{18}$ It may prove possible to obtain an integrated measure (or measures) of domain-specific cognition from such work. The construction of measures of domain-specific affect is also a task for the future. Here the tradition of research on job satisfaction affords many suggestions, though it also presents problems, and does not provide any immediately available measures in which cognition and affect are covered separately. Although the concepts of cognition and affect have a considerable intellectual history within psychology (McKennell, 1978), investigators of subjective well-being must look to their own research to clarify the distinction as it applies to their own field.

It is hoped that the present model-building approach will contribute to the development of theory in quality of life research. The approach forces a level of specificity in theoretical thinking which is not often achieved in the absence of a specific model. Above all, the models have heuristic value. They make explicit the logical consequences of the assumptions underlying theoretical formulations, and where information is not yet available for testing a crucial model, the very exercise of specifying that model serves to identify the precise type of information that is needed, thereby pointing the direction in which future research should go.

University of Southampton, England

University of Michigan, U.S.A.

\section{NOTES}

1 The investigations reported in this paper were supported through grant Number SOC77-06525 from the National Science Foundation. We are grateful to Mary Grace Moore for valuable assistance in the preparation of computer runs, and to Suzanne Gurney for skillful typing. Willard Rodgers provided helpful comments on a previous draft of this report.

2 Models of this form are sometimes referred to as 'confirmatory' or 'restricted' factor analysis models (e.g., Joreskog, 1969; Boruch and Wolins, 1970). For further discussion of 
the application of this form of the LISREL model to the estimation of the reliabilities and validities of measures of well-being, see Andrews and Withey (1976, Chapter 6), also McKennell et al, (1978). The more general LISREL procedure is sometimes referred to as 'second-order confirmatory factor analysis' (Burt, 1973).

${ }^{3}$ According to Long (1976, pp. 171-172), chi-square can be applied as a test statistic only for the differentiation between 'nested' models. Models are nested when the one with fewer free parameters (higher degrees of freedom, $d_{0}$ ) has no parameters other than those present in the less restrictive model (degrees of freedom $d_{1}$ ). Under these conditions the difference between the chi-square values is itself distributed as chi-square with degrees of freedom equal to $d_{0}-d_{1}$. However, in our experience, even where these conditions are met, the probability levels associated with differences in chi-square are of limited usefulness when models are estimated using data from relatively large numbers of respondents. Modifications in the direction of parsimony tend to be ruled out simply because the more complex model (that fits more parameters and that has fewer degrees of freedom) usually yields a smaller chi-square, and almost invariably, with large samples, the difference will be statistically significant. One is again led back to the descriptive or exploratory use of the chi-square statistic in which changes in chi-square are assessed qualitatively in relation to the shift in degrees of freedom.

4 The results of a confirmatory factor analysis of items from Bradburn's (1969) affect scales together with five indicators of global well-being have been presented previously by McKennell (1978, Table III). The model in Exhibit 2a differs from this earlier one in that the correlation between the cognitive and the affect factors has been fixed at zero, the loadings on the method factor have been fixed at values ascertained in separate analyses (Andrews and McKennell, 1978, Exhibit 3), a six th global indicator - the Thermometer scale - has been introduced, and the affect scales rather than their constituent items have been used as inputs (see Andrews and McKennell, 1978, Section 5.1, especially Notes 6 and 7). Throughout this present paper a decision has been made to simplify the relationships between the affect and cognitive factors by treating them as orthogonal; however, elsewhere we have described some effects of allowing these factors to be correlated (Andrews and McKennell, 1978, Section 5.3).

5 A few, not very extensive, replications were made of the comparisons between the affect-cognition and affect-LAW models in Figures $2 a$ and $2 b$ for subgroups of the total sample. Comparisons were made for two small subgroups: people with high socioeconomic status (SES) who were aged thirty or over $(N=140)$, and low SES people aged thirty or over $(N=100)$. For these small subgroups it was found that although there was a decrease in Chi-square on moving from the affect-LAW to the affect-cognition model, the saving in chi-square points was not greater than the drop in degrees of freedom. A similar comparison between models was also made for two large subgroups: Males $(N=440)$ and females $(N=580)$. For females the decrease in chi-square points (36) was substantially greater than the saving in degrees of freedom (10); for males the decrease (12) was only slightly greater than the saving in degrees of freedom (10). While the results for the two larger subgroups, particularly the females, replicates the result in the total sample that favored the affect-cognition model, the results for the two smaller subgroups did not. However, these small subgroups are particularly subject to sampling instability and the sensitivety of the chi-square difference test to sample size.

- For convenience in later graphical displays, this paper will use 'domain' to refer to all types specific life concerns. This usage differs from that of Andrews and Withey (1976) who distinguished two types of life concerns - domains and criteria.

Burt et al. (1978) have argued that only one satisfaction with domains factor is necessary to model the structure of well-being. We have preferred to use several domain factors, however, on the following grounds: The set of fifteen domains considered in this paper was designed to sample the perceptual space established by a comprehensive mapping of domains (see Andrews and Withey, 1976, Chapter 2); factor analyses of an 
adequate set of domains have previously established the existence of several factors (see Andrews and Withey, 1976, Exhibit E.2); the chi-square value of 2255 for 229 degrees of freedom that was obtained for a model fitted to only one summed-domain factor compared very poorly to the fit obtained with a comparable model in which the domains were decomposed into factors (i.e., in the model of Exhibit 6); the separate domain factors correlate differentially in substantively interesting ways with positive and negative affect; and, finally, it makes substantive sense to make room in our models for the possibility that different individuals or groups or cultures may vary in the weighting given to separate domain factors.

7 The correlations with the affect scales of the items in domain factors 2,3 , and 4 - as designated in Figure $3 a$ - are, respectively, on average, $0.24,0.13$ and 0.12 . Correlations with positive and negative affect were about equal in strength (but opposite in sign). For the items on domain factor 1 , on the other hand, the average correlation with positive affect was 0.27 and with negative affect 0.21 . For factor 5 , the corresponding average correlations were 0.08 and 0.19 .

- McKennell also discussed and made tentative parameter estimates for a simplified model that placed affect measures in an exogenous position while using a single composite variable, $\Sigma C$, the summed domain evaluations, to represent the domains (see McKennell, 1978, Figure 7). In the course of the work leading to the present paper, a more complex version of this model was run which included the life-as-a-whole factor and a method factor as well as a summed-domain factor. The obtained parameter values were very near those estimated by McKennell. This model was run first with paths from affect to the LAW factor ( 8 paths) again with these paths deleted. As has been found consistently, deletion of the $g$ paths did not increase the residual coefficient for the prediction of the LAW factor.

9 This result accords with the finding from regression runs with the observed variables (see the discussion relative to Table IV in McKennell, 1978). There is an apparent anomaly in the comparison between Models 6A and 6B (which occurs again in the comparison of Models $6 \mathrm{C}$ and $6 \mathrm{D}$ ) in that adding in affect as a predictor appears to lead to a small increment in the size of the residual coefficient for LAW. This is believed to result from minute changes in the definition of the constituent factors in the model. It should be stressed that these changes are very small (visible only as shifts here and there in the second decimal place of the loadings on some of the factors). The changes in the residual coefficients are of approximately the same order as their standard errors. The correct inference therefore appears to be one of no difference between these residual coefficients.

${ }_{10}$ See Note 6 for the averaged correlations of the items in the factors with the affect scales. The correlations for the factors in Exhibit 8 are higher due to the correction for random error, but the pattern of correlation is preserved.

${ }_{11}$ The model of Exhibit 11 is an implementation of the model in Figure 6 in McKennell (1978).

${ }_{12}$ Two other kinds of path models containing a cognitive factor were also examined. In both of these the cognitive factor was made the endogenous variable being predicted. The first was the path analogue of the model shown in Exhibit 3a. The second was a kind of hybrid mid-way between the latter and model 6C (considered in Exhibit 7, but without the $g$ path), in that affect was not factored out of the domains but only out of the global indicators. Both these trial models fitted the data fairly well. (Chi-square equalled 507 with 178 degrees of freedom on the first case and 562 with 197 degrees of freedom in the second.) However, both were rejected for several reasons. First, on the ground of external validity: The residual coefficient for the prediction of the cognitive factor was high, 0.48 in the first case and 0.32 in the second; These values compared unfavourably with the 0.24 coefficient obtained for Model 6C in Exhibit 7. Secondly, 
because in the hybrid model the loadings of the global indicators on the cognitive factor changed from those found previously, thereby altering the meaning of this factor. Thirdly, and perhaps most important, because it was not clear what the substantive interpretation of either of these models could be in causal terms.

13 Variables have been reversed to provide natural scoring.

14 The British data does not allow much analysis of the way observed measures relate to their underlying factors. However, the broad structural constancies under discussion can be added to other evidence derived from the analysis of data on life quality from Englishand French-speaking Canadians (McKennell et al., 1978), which suggests that similar measurement procedures, at least when applied by efficient survey organisations, will yield measures with much the same error and validity components despite cultural and even language differences.

is For the Abrams-Hall data the first four domains to enter the stepwise regression, in order of entry, were Standard of Living (0.23), Leisure (0.22), Finance (0.16), and Family Life (0.12) (final Beta coefficients in brackets). For the Andrews-Withey data the order was Family Life (0.29), Standard of Living (0.16), Job (0.13), and Spare Time Activities (0.13). These four domains accounted for 47 per cent of the variance in the LAW measure in the Abrams-Hall data and 53 per cent in the Andrews-Withey data.

16 In the NORC data set (Burt et al., 1978) the $R^{2}$ for the prediction of positive and negative affect from the domain measures was 0.05 and 0.08 respectively. For the prediction of the global satisfaction measure from affect and the domains the $R^{2}$ was 0.30 . The first four domains to enter into the stepwise prediction of global satisfaction (after forcing in affect) were Leisure (0.22), Finance (0.17), Housing $(0.10)$ and Work (0.13) in order of entry (final Beta coefficients in brackets).

${ }_{17}$ An attempt to replicate the factor model published by Burt et al., (1978) was abandoned when it was discovered that this model was not identified (see Burt et al., (1978). A comparison of an affect-LAW versus an affect-cognition model, along the lines of Exhibits $2 b$ and $2 a$, for the NORC data did not lead to an improvement in fit for the affect-cognition model. However, since only two global indicators were available for defining the cognitive factor, it was decided not to pursue the analysis of cognition further with these data.

18 Personal Communications.

\section{BIBLIOG R A PHY}

Abrams, M.: 1975, Subjective Measures of Perceived Injustices, Paper presented at the Conference on Subjective Measures of Quality of Life, jointly sponsored by the British and American Social Science Research Councils, Cambridge, England.

Abrams, M.: 1976, A Review of Work on Subjective Social Indicators: 1971-1975 (Social Science Research Council Survey Unit, London).

Andrews, F.M. and McKennell, A.C.: 1978, Measures of Self-reported Well-being: Their Affective, Cognitive and Other Components (Institute for Social Research, Unpublished document, Ann Arbor, Michigan).

Andrews, F.M. and Withey, S.B.: 1976, Social Indicators of Well-being (Plenum Press, New York).

Bradburn, N.M.: 1969, The Structure of Psychological Well-being (Aldine, Chicago).

Bortner, R.W. and Hultsch, F.D.: 1973, 'Patterns of subjective deprivation in adulthood', Development Psychology 10, pp. 534-545.

Boruch, R.F. and Wolins, L.: 1970, 'A procedure for estimation of trait, method, and error variance attribute to a measure', Educational and Psychological Measurement 30 , pp. $547-574$. 
Burt, R.S.: 1973, 'Confirmatory factor analy tic structures and the theory construction process', Sociological Methods and Research 2, pp. 131-190.

Burt, R.C., Fischer, M.G., and Christman, K.P.: 1978, A Note on Sufficient Conditions for Identifying Population Structures of Well-being as Restricted Covariance Models (University of California, Survey Research Center Working Paper 9, Berkeley, California).

Burt, R.S., Wiley, J.A., Minor, M.J., and Murrey, J.R.: 1978, 'Structure of well-being: Form, content, and stability over time', Sociological Methods and Research, pp. $365-407$.

Campbell, A., Converse, P.E., and Rodgers, W.L.: 1976, The Quality of American Life: Perceptions, Evaluations, and Satisfactions (Russell Sage Foundation, New York).

Duncan, O.D.: 1966, 'Path analysis: Sociological examples', American Journal of Sociology 72, pp. 1-16.

Goldberger, A.S.: 1972, 'Structural equation methods in the social sciences', Econometrica 40, pp. 979-1001.

Goldberger, A.S. and Duncan, O.D. (eds.): 1974, Structural Equation Models in the Social Sciences (Seminar Press, New York).

Hall, J.: 1975, The Relationship Between Subjective and Objective Indicators of Individual Well-being - A Linear Modelling Approach, Paper presented to the joint seminar on 'Subjective measures of quality of life' sponsored by the Social Science Research Councils of Britain and U.S.A., Cambridge, England.

Hall, J.S.: 1976, 'Subjective measures of quality of life in Britain: 1971 to 1975 - Some developments and trends', Social Trends No. 7 (Her Majesty's Stationery Office, London).

Harman, H.H.: 1976, Modern Factor Analysis (University of Chicago Press, Chicago).

Joreskog, K.G.: 1969, 'A general approach to confirmatory maximum likelihood factor analysis', Psychometrika 34, pp. 183-202.

Joreskog, K.G.: 1970, 'A general method for analysis of covariance structures', Biometrika 57, pp. 239-251.

Joreskog, K.G.: 1973, 'A general method for estimating a linear structural equation system', In Structural Equation Models in the Social Sciences, ed. by A. Goldberger and O.D. Duncan (Seminar Press, New York), pp. 85-112.

Joreskog, K.G. and Sorbom, D.: 1970, LISREL III User's Guide (National Educational Resources, Chicago).

Long, J.S.: 1976, 'Estimation and hypothesis testing in linear models containing measurement error: A review of Joreskog's model for the analysis of covariance structures', Sociological Methods and Research 5, pp. 157-206.

Mason, R. and Faulkenberry, G.D.: 1978, 'Aspirations, achievements, and life satisfaction', Social Indicators Research 5, pp. 133-150.

McKennell, A.C.: 1978, 'Cognition and affect in perceptions of well-being', Social Indicators Research 5, pp. 389-426.

McKennell, A.C., Atkinson, T., and Andrews, F.M.: 1978, Structural Constancies in Surveys of Perceived Well-being, Paper delivered at 9th World Congress of Sociology, Uppsala, Sweden. 